Hui $\mathrm{Li}$

Lizhu Hao

Huilong Ren

Bo Tian

http://dx.doi.org/10.21278/brod67306

ISSN 0007-215X

eISSN 1845-5859

\title{
THE APPLICATION OF MESHLESS CYLINDER CONTROL SURFACE IN RANKINE-KELVIN HYBRID METHOD
}

UDC 629.5.015.2:629.5.017.2:519.61

Original scientific paper

\begin{abstract}
Summary
The solution of the three-dimensional seakeeping problem with forward speed in the frequency domain still has some well-known problems. In this paper, a Rankine-Kelvin hybrid method benefiting the merits of both the Rankine source and Kelvin source is presented and demonstrated by studying the wave diffraction/radiation problem with zero forward speed as an example. A meshless cylindrical surface is selected to be the control surface dividing the fluid domain into two regions, and the velocity potential and its normal derivative on the control surface are represented by series expansions. The present RankineKelvin hybrid method is validated by the added mass and damping associated with the linear radiation force, and comparison is made with the documented analytical solution. All the work in the paper sheds light on solving the forward speed hydrodynamic problems.
\end{abstract}

Key words: Rankine-Kelvin hybrid method; meshless cylindrical control surface; series expansion method;

\section{Introduction}

As it is known, when solving the hydrodynamic problem with forward speed, the implementation of the Kelvin source method using the Green function associated with a translating and oscillating source bring along several well-known problems concerning its wave component. In particular, it behaves with complex singularities and high oscillations when both the field and source points approach to the free surface [1]. In the earlier work, numerical results were in very good agreement for immersed bodies like sphere and ellipsoid, or surface piecing body of very slender shape like Wigley hull [2]. Unfortunately, for the realistic container ships, the results present large discrepancy from those of model tests. Ten and Chen (2010) [3] came up with a new method to solve the forward speed problem, and 
they try to benefit the merits of the Rankine source method and the Kelvin source method by using a hemisphere as a control surface. However, the method made the calculation of the integration over the control surface complex and may lead to some troubles about singularities.

In this work, we use a cylinder as a control surface instead of a semi-sphere in the same spirit as Liang and Chen's work[4]. The Rankine source method is used in the internal domain, and the fundamental solution is in the form of $1 / r$, with $r$ standing for the distance between the field point and the source point. Panels are distributed over the body surface and the free surface in the internal domain. Kelvin source method is used in the external domain and the Green function which satisfies the free-surface boundary condition and radiation condition is adopted. On the meshless cylindrical control surface, the velocity potential and its normal derivative are expanded into Fourier-Laguerre series and Lv et al. [5] have proved the effectiveness of approximation by Laguerre series for diffraction waves on the cylinder.

The objective of this work is to solve the zero speed seakeeping problem as an example to show the effectiveness of Rankine-Kelvin hybrid method with a meshless cylindrical control surface. In the numerical implementation, we consider a hemisphere floating at the free surface, and the forward speed is out ruled at the moment. The added mass and damping coefficients are calculated with the present Rankine-Kelvin hybrid method, and the convergence test associated with the terms of Laguerre functions and Fourier series is made. In addition, the influence of different control surface radius on the numerical results is studied through comparing with the analytical solution, and eventually a satisfactory accuracy is obtained. Due to the fact that there are many new defined coefficients concerning multi-fold integrals in this method, Chebyshev expansions are utilized to approximate the resultant integral to improve computational efficiency. The successful application of the RankineKelvin hybrid method in zero speed problem has established a foundation for solving the forward speed problem.

\section{The series expansion method of velocity potential and its normal derivative}

In the Rankine-Kelvin hybrid method, as a meshless circular cylinder surface is selected as control surface, the velocity potential and its normal derivative on the control surface need to be expressed analytically. In this paper, they are expanded into Fourier-Laguerre series.

The velocity potential on the control surface is a function associated with the polar angle $\varphi$ and vertical coordinate $z$ positively downward, which can be expressed as $\phi(\varphi, z)$. As the velocity potential $\phi(\varphi, z)$ decreases exponentially with the increase of $\mathrm{z}$, and when $\mathrm{z}$ tends to infinity, the velocity potential tends to zero. The velocity potential is expanded into series of Laguerre as follows

$$
\phi(\varphi, z)=\sum_{n=0}^{+\infty} f_{n}(\varphi) L_{n}(z) e^{-\frac{z}{2}}=\sum_{n=0}^{+\infty} f_{n}(\varphi) \ell_{n}(z)
$$

where $L_{n}(z)$ is $\mathrm{n}$-th order Laguerre polynomial, and $\ell_{n}(z)=L_{n}(z) e^{-\frac{z}{2}}$ is the $\mathrm{n}$-th order Laguerre function.

The orthogonal property of Laguerre polynomials is considered in Eq. (2) and Eq. (3).

$$
\int_{0}^{+\infty} L_{k}(z) L_{n}(z) e^{-z} d z=\delta_{n k}
$$




$$
\int_{0}^{+\infty} \ell_{k}(z) \ell_{n}(z) d z=\delta_{n k}
$$

where $\delta_{n k}$ is Kronecker delta function.

Thus, $\ell_{n}(z)$ is multiplied on the two sides of the Eq. (1) simultaneously and we can integrate the both sides from zero to infinity with respect to $z$, and the coefficient of the series expansion can be expressed as follows

$$
f_{n}(\varphi)=\int_{0}^{+\infty} \phi(\varphi, z) \ell_{n}(z) d z
$$

As the velocity potential on the control surface is a periodic function about $\varphi$ of which the period is $2 \pi$, so we have $\phi(\varphi, z)=\phi(\varphi+2 \pi, z), f_{n}(\varphi)=f_{n}(\varphi+2 \pi)$ and the Dirichlet condition is satisfied on the period $(-\pi, \pi)$, namely

1. The function is continuous or has finite discontinuity points of the first kind.

2. The function has finite extreme points.

So the velocity potential on the control surface can be expanded into Fourier series, and its complex exponential form is as follows

$$
f_{n}(\varphi)=\sum_{m=-\infty}^{+\infty} \phi_{n m} e^{i m \varphi}
$$

with

$$
\phi_{n m}=\frac{1}{2 \pi} \int_{-\pi}^{\pi} f_{n}(\varphi) e^{-i m \varphi} d \varphi
$$

The substitution Eq. (5) into Eq. (1) yields

$$
\phi(\varphi, z)=\sum_{m=-\infty}^{+\infty} \sum_{n=0}^{+\infty} \phi_{n m} \ell_{n}(z) e^{i m \varphi}
$$

The substitution Eq. (4) into Eq. (6) yields

$$
\phi_{n m}=\frac{1}{2 \pi} \int_{0}^{+\infty} \int_{-\pi}^{\pi} \phi(\varphi, z) \ell_{n}(z) e^{-i m \varphi} d \varphi d z
$$

In the same way, the normal derivative of velocity potential $\psi(\varphi, z)$ of an arbitrary point on the control surface can be also expanded into Laguerre-Fourier series as follows

$$
\begin{aligned}
& \psi(\varphi, z)=\sum_{m=-\infty}^{+\infty} \sum_{n=0}^{+\infty} \psi_{n m} \ell_{n}(z) e^{i m \varphi} \\
& \psi_{n m}=\frac{1}{2 \pi} \int_{0}^{+\infty} \int_{-\pi}^{\pi} \psi(\varphi, z) \ell_{n}(z) e^{-i m \varphi} d \varphi d z
\end{aligned}
$$

In (7) and (9), $\phi_{n m}$ and $\psi_{n m}$ are coefficients of the series expansion and defined by (8) and (10), respectively. 


\section{The application of rankine-kelvin hybrid method to zero speed problem}

\subsection{Definition of parameters}

We consider the ship floating in the sea of infinite depth with zero speed and use a cylinder to separate the whole fluid domain into two domains. In the external domain, the normal vectors of the boundary surfaces positively inward to the domain. In the internal domain, the normal vectors point positively outwards of the domain. The normal vectors and the coordinate system are shown in Figure 1.



Fig. 1 Definition of the coordinate system and normal vectors

It is convenient to use the cylindrical coordinate system as a cylindrical surface is chosen to be the control surface. Here is the transformation between the two coordinates.

$$
x=h \cos \varphi \quad y=h \sin \varphi \quad z=\zeta
$$

where $h$ is the radius of the cylinder, $\varphi \in(-\pi, \pi)$ varies in the plane $x o y, \zeta \in(0,+\infty)$, and then an arbitrary point can be expressed as $P(h, \varphi, z)$ in the cylindrical coordinate system.

The boundary conditions in the internal domain are given as followings

$$
\begin{array}{ll}
\frac{\partial \phi}{\partial n}=-\frac{\partial \phi}{\partial z}=\frac{\omega^{2}}{g} \phi & \text { on the free surface } z=0 \\
\frac{\partial \phi_{j}}{\partial n}=n_{j}(j=1,2, \cdots, 6) & \text { on the body surface }
\end{array}
$$

where $\omega$ denotes the oscillation frequency.

\subsection{External problem}

In the external domain, the Green function used here is given in the form of [6]

$$
G(P, \mathrm{Q})=\frac{1}{r_{P \mathrm{Q}}}+\frac{1}{r_{P \overline{\mathrm{Q}}}}+2 v_{0} P . V \cdot \int_{0}^{+\infty} \frac{1}{v-v_{0}} e^{-v(z+\zeta)} J_{0}(v R) d v+i 2 \pi v_{0} e^{-v_{0}(z+\zeta)} J_{0}\left(v_{0} R\right)
$$

In which, $v_{0}=\omega^{2} / g$ is the wave number, $J_{0}()$ is the zeroth order Bessel function of the first kind, P.V. is principle-value integral.

For the velocity potential at an arbitrary field point $P$ on the control surface in the external domain, application of the Green's second identity provides

$$
2 \pi \phi(P)=\int_{c}\left[\phi(\mathrm{Q}) \frac{\partial}{\partial n_{\mathrm{Q}}} G(P, \mathrm{Q})-G(P, \mathrm{Q}) \frac{\partial}{\partial n_{\mathrm{Q}}} \phi(\mathrm{Q})\right] d s_{\mathrm{Q}}
$$


After substituting Eqs. (7) and (9) into Eq. (15), we obtain

$$
2 \pi \sum_{l=-\infty}^{+\infty} \sum_{k=0}^{+\infty} \phi_{k l} \ell_{k}(z) e^{i l \varphi}=\int_{c}\left[\frac{\partial}{\partial n_{\mathrm{Q}}} G \sum_{l=-\infty}^{+\infty} \sum_{k=0}^{+\infty} \phi_{k l} \ell_{k}(\zeta) e^{i l \varphi^{\prime}}-G \sum_{l=-\infty}^{+\infty} \sum_{k=0}^{+\infty} \psi_{k l} \ell_{k}(\zeta) e^{i l \varphi^{\prime}}\right] d s
$$

Application of Eq. (8), we have

$$
2 \pi \phi_{n m}=\frac{1}{2 \pi} \int_{0}^{+\infty} \int_{-\pi}^{\pi} \int_{c}\left[\frac{\partial}{\partial n_{Q}} G \sum_{l=-\infty}^{+\infty} \sum_{k=0}^{+\infty} \phi_{k l} \ell_{k}(\zeta) e^{i l \varphi^{\prime}}-G \sum_{l=-\infty}^{+\infty} \sum_{k=0}^{+\infty} \psi_{k l} \ell_{k}(\zeta) e^{i l \varphi^{\prime}}\right] \ell_{n}(z) e^{-i m \varphi} d s d \varphi d z
$$

We define new coefficients

$$
\begin{aligned}
& G_{n m, k l}=\frac{1}{2 \pi} \int_{0}^{+\infty} \int_{-\pi}^{\pi} \int_{c} G \ell_{k}(\zeta) \ell_{n}(z) e^{i l \varphi^{\prime}} e^{-i m \varphi} d s d \varphi d z \\
& H_{n m, k l}=\frac{1}{2 \pi} \int_{0}^{+\infty} \int_{-\pi}^{\pi} \int_{c} \frac{\partial}{\partial n_{\mathrm{Q}}} G \ell_{k}(\zeta) \ell_{n}(z) e^{i l \varphi^{\prime}} e^{-i m \varphi} d s d \varphi d z
\end{aligned}
$$

So Eq. (17) can be rewritten as

$$
2 \pi \phi_{n m}=\sum_{l=-\infty}^{+\infty} \sum_{k=0}^{+\infty} H_{n m, k l} \phi_{k l}-\sum_{l=-\infty}^{+\infty} \sum_{k=0}^{+\infty} G_{n m, k l} \psi_{k l}
$$

As the subscripts $n$ and $m$ change, we can get a system of equations which can be expressed as follow

$$
\left(H^{c, c}-2 \pi E_{1}\right) \vec{\phi}_{k l}=G^{c, c} \vec{\psi}_{k l}
$$

Here $H^{C, C}, G^{C, C}$ are matrices consisted of $H_{n m, k l}$ and $G_{n m, k l}$, and $\vec{\phi}_{k l}, \vec{\psi}_{k l}$ are vectors consisted of $\phi_{k l}$ and $\psi_{k l}, E_{1}$ is the unit matrix of which the dimension is equal to $H^{C, C}$.

Finally we can get the relationship between the series expansion coefficients of the velocity potential and its normal derivative on the control surface.

$$
\vec{\psi}_{k l}=\left(G^{C, C}\right)^{-1}\left(H^{C, C}-2 \pi E_{1}\right) \vec{\phi}_{k l}
$$

And we define the matrix DN as

$$
D N=\left(G^{C, C}\right)^{-1}\left(H^{C, C}-2 \pi E_{1}\right)
$$




\subsection{Extended boundary integral equation method}

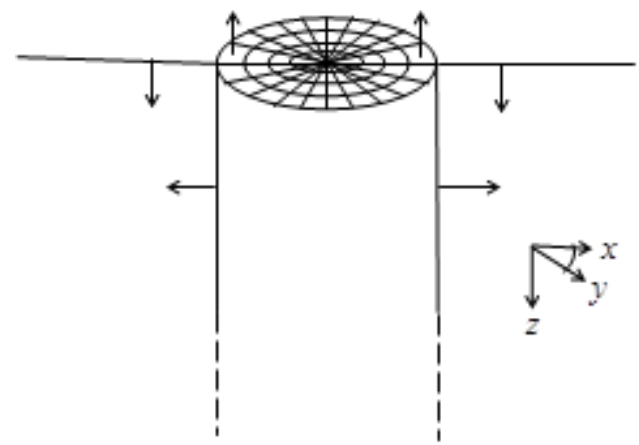

Fig. 2 Schematic diagram of extended boundary integral equation method

As the application of the free-surface Green function in the external domain problem will result in the occurrence of irregular frequencies, extended boundary integral equation method presented in [7] is adopted to remove irregular frequencies, and the interior free surface is divided into panels shown in Figure 2. The boundary integral equations are given as follows

$$
\begin{array}{ll}
2 \pi \phi(P)+\int_{c} G \frac{\partial}{\partial n_{\mathrm{Q}}} \phi(\mathrm{Q}) d s+\int_{F} \mu \frac{\partial}{\partial n_{\mathrm{Q}}} G d s=\int_{c} \phi(\mathrm{Q}) \frac{\partial}{\partial n_{\mathrm{Q}}} G d s \quad P \in C \\
4 \pi \mu+\int_{c} \phi(\mathrm{Q}) \frac{\partial}{\partial n_{\mathrm{Q}}} G d s+\int_{F} \mu \frac{\partial}{\partial n_{\mathrm{Q}}} G d s=\int_{c} G \frac{\partial}{\partial n_{\mathrm{Q}}} \phi(\mathrm{Q}) d s & P \in F
\end{array}
$$

The dipole distribution $\mu$ on each panel of the interior free surface is assumed constant. Substituting Eqs. (7) and (9) into Eqs. (24) and (25) and applying Eq. (8), we can get the following integral equations.

When field point is on control surface

$$
2 \pi \phi_{n m}=\sum_{l=-\infty}^{+\infty} \sum_{k=0}^{+\infty} H_{n m, k l} \phi_{k l}-\sum_{l=-\infty}^{+\infty} \sum_{k=0}^{+\infty} G_{n m, k l} \psi_{k l}-\sum_{i=1}^{N_{F}} H_{n m, F} \mu_{i}
$$

with

$$
H_{n m, F}=\frac{1}{2 \pi} \int_{0}^{+\infty} \int_{-\pi}^{\pi} \int_{\Delta F_{i}} G_{n} \ell_{n}(z) e^{-i m \varphi} d s d \varphi d z
$$

$G_{n m, k l}$ and $H_{n m, k l}$ have been defined in Eqs. (18) and (19).

When field point is on interior free surface

$$
4 \pi \mu_{i}+\sum_{l=-\infty}^{+\infty} \sum_{k=0}^{+\infty} H_{F, k l} \phi_{k l}-\sum_{l=-\infty}^{+\infty} \sum_{k=0}^{+\infty} G_{F, k l} \psi_{k l}+\sum_{i=1}^{N_{F}} H_{F, F} \mu_{i}=0
$$

with

$$
\begin{aligned}
& H_{F, k l}=\int_{c} G_{n} \ell_{k}(\zeta) e^{i l \varphi^{\prime}} d s \\
& G_{F, k l}=\int_{c} G \ell_{k}(\zeta) e^{i l \varphi^{\prime}} d s
\end{aligned}
$$




$$
H_{F, F}=\int_{\Delta F_{i}} G_{n} d s
$$

In a similar way with Eq. (21), we can get a matrix form

$$
\left[\begin{array}{ll}
H^{C, C}-2 \pi E_{1} & -H^{C, F} \\
H^{F, C} & H^{F, F}+4 \pi E_{2}
\end{array}\right]\left[\begin{array}{l}
\vec{\phi}_{k l} \\
\vec{\mu}
\end{array}\right]=\left[\begin{array}{l}
G^{C, C} \\
G^{F, C}
\end{array}\right] \vec{\psi}_{k l}
$$

Among them, $H^{C, F}, H^{F, C}, G^{F, C}, H^{F, F}$ are separately consisted of $H_{n m, F}, H_{F, k l}, G_{F, k l}, H_{F, F}$, and the vector $\vec{\mu}$ is consisted of $\mu_{i} . E_{2}$ is the unit matrix of which the dimension is equal to $H^{F, F}$.

From Eq. (32), we can get the new relationship between the series expansion coefficients of the velocity potential and its normal derivative on the control surface

$$
\vec{\psi}_{k l}=\overline{D N} \vec{\phi}_{k l}
$$

among them

$$
\begin{aligned}
\overline{D N}= & {\left[G^{C, C}+H^{C, F}\left(4 \pi E+H^{F, F}\right)^{-1} G^{F, C}\right]^{-1} } \\
& \cdot\left[\left(H^{C, C}-2 \pi E\right)+H^{C, F}\left(4 \pi E+H^{F, F}\right)^{-1} H^{F, C}\right]
\end{aligned}
$$

\subsection{The validation of the external domain}

To verify the relationship between the velocity potential and its normal derivative obtained from the external domain, we have calculated the diffraction potential of an infinitely long vertical circular cylinder.

We consider a regular wave as the incident wave, which propagates along the positive axis of $x$, and the corresponding first order incident wave potential is as follows

$$
\phi_{0}=\frac{g A}{i \omega} e^{-v_{0} z} e^{i v_{0} x}=\frac{g A}{i \omega} e^{-v_{0} z} \sum_{m=0}^{\infty} \varepsilon_{m} i^{m} J_{m}\left(v_{0} R\right) \cos m \varphi
$$

(when $m=0, \varepsilon_{m}=1$, or $\varepsilon_{m}=2$ )

Among them, $A$ is the amplitude of the incident wave, and $(R, \varphi, z)$ is the cylindrical coordinates of an arbitrary point. According to the body surface condition, we can get

$$
\frac{\partial}{\partial n} \phi_{7}=-\frac{\partial}{\partial n} \phi_{0}=-\frac{\partial}{\partial R} \phi_{0}=-\frac{g A}{i w} e^{-v_{0} z} \sum_{m=0}^{+\infty} \varepsilon_{m} i^{m} J_{m}^{\prime}\left(v_{0} R\right) v_{0} \cos m \varphi
$$

Applying Eqs.(9) and (10), the series expansion coefficient of the normal derivative of diffraction potential can be obtained 


$$
\begin{aligned}
\psi_{k l} & =\frac{1}{2 \pi} \int_{-\pi}^{\pi} \int_{0}^{+\infty}\left[-\frac{g A}{i w} e^{-v_{0} z} \sum_{m=0}^{+\infty} \varepsilon_{m} i^{m} J_{m}^{\prime}\left(v_{0} R_{0}\right) v_{0} \cos m \varphi\right] \ell_{k}(z) e^{-i l \varphi} d z d \varphi \\
& =-\frac{g A}{i w} \frac{\left(2 v_{0}-1\right)^{k}}{\left(2 v_{0}+1\right)^{k+1}} \sum_{m=0}^{+\infty} \varepsilon_{m} i^{m} J_{m}^{\prime}\left(v_{0} R_{0}\right) v_{0} \tau_{m|l|}
\end{aligned}
$$

where

$$
\tau_{m|l|}= \begin{cases}2 & (m=|l|=0) \\ \delta_{m|l|} & (\text { others })\end{cases}
$$

Appendix 1 can be referred for the calculation details of Eq. (37)

Substitute Eq. (37) into Eq. (22) and (33), we can get the series expansion coefficients of the diffraction potential, then numerical solution can be obtained based on Eq. (7).

At the same time, the diffraction force of an infinitely long vertical circular cylinder $F_{w j}^{d}$ can be calculated based on the following Eq.

$$
F_{w j}^{d}=\rho i w \iint_{s_{0}} \phi_{7} n_{j} d s \quad(j=1,2,3)
$$

Substitute Eq. (7) into Eq. (39), then

$$
F_{w j}^{d}=\rho i w R_{0} \sum_{m=-\infty}^{+\infty} \sum_{n=0}^{+\infty} \phi_{n m} \int_{0}^{+\infty} \int_{-\pi}^{\pi} \ell_{n}(z) e^{i m \varphi} n_{j} d z d \varphi
$$

Substitute $\vec{n}=(-\cos \varphi,-\sin \varphi, 0)$ into Eq. (40), It is obvious that $F_{w 2}^{d}=0, F_{w 3}^{d}=0$,

$$
F_{w 1}^{d}=-2 \pi \rho i w R_{0} \sum_{n=0}^{+\infty}(-1)^{n}\left(\phi_{n, 1}+\phi_{n,-1}\right)
$$

Analytical solution of the diffraction potential [6] is as follow

$$
\varphi_{7}=-\frac{g A}{i w} e^{-v_{0} z} \sum_{m=0}^{\infty} \varepsilon_{m} i^{m} \frac{J_{m}^{\prime}\left(v_{0} R_{0}\right)}{H_{m}^{\prime}\left(v_{0} R_{0}\right)} H_{m}\left(v_{0} R\right) \cos m \varphi
$$

Substitute Eq. (42) into Eq. (39), analytical solution to the diffraction force can be expressed as follow

$$
F_{w 1}^{d}=\frac{\rho g R_{0} A}{v_{0}} \varepsilon_{1} i \frac{J_{1}^{\prime}\left(v_{0} R_{0}\right)}{H_{1}^{\prime}\left(v_{0} R_{0}\right)} H_{1}\left(v_{0} R_{0}\right) \pi
$$

Comparison of the numerical solutions for the diffraction potential and diffraction force with the analytical ones are presented in Section 4.

\subsection{Internal problem}

In the internal domain, Rankine source is adopted which is given as follow

$$
G_{r}=\frac{1}{r_{P Q}}
$$


For the velocity potential at an arbitrary field point $\mathrm{P}$ on the boundary surface of internal domain consisting of control surface $S_{C}$ free surface $S_{F}$ and body surface $S_{B}$, application of the Green's second identity yields

$$
2 \pi \phi(P)=\int_{S_{C}+S_{B}+S_{F}}\left[G_{r} \frac{\partial \phi(\mathrm{Q})}{\partial n_{\mathrm{Q}}}-\phi(\mathrm{Q}) \frac{\partial}{\partial n_{\mathrm{Q}}} G_{r}\right] d s_{\mathrm{Q}}
$$

The free surface and body surface are divided by $N_{B}$ and $N_{F}$ panels, respectively. Thus, expression (45) becomes

$$
2 \pi \phi(P)=\left(\sum_{\alpha=1}^{N_{B}} \int_{B^{\alpha}}+\sum_{\beta=1}^{N_{F}} \int_{F^{\beta}}+\int_{C}\right)\left[G_{r} \frac{\partial \phi(\mathrm{Q})}{\partial n_{\mathrm{Q}}}-\phi(\mathrm{Q}) \frac{\partial}{\partial n_{\mathrm{Q}}} G\right] d s
$$

Substitute the boundary conditions Eqs. (12) and (13) into Eq. (46),

$$
\begin{aligned}
& 2 \pi \phi(P)=\int_{c}\left[G_{r} \frac{\partial \phi(\mathrm{Q})}{\partial n_{\mathrm{Q}}}-\phi(\mathrm{Q}) \frac{\partial}{\partial n_{\mathrm{Q}}} G_{r}\right] d s+\sum_{\alpha=1}^{N_{B}} \int_{B^{\alpha}} G_{r} n_{\alpha} d s-\sum_{\alpha=1}^{N_{B}} \int_{B^{\alpha}} \phi(\mathrm{Q}) \frac{\partial}{\partial n_{\mathrm{Q}}} G_{r} d s \\
& +\frac{\omega^{2}}{g} \sum_{\beta=1}^{N_{F}} \int_{F^{\beta}} G_{r} d s-\sum_{\beta=1}^{N_{F}} \int_{F^{\beta}} \phi(\mathrm{Q}) \frac{\partial}{\partial n_{\mathrm{Q}}} G_{r} d s
\end{aligned}
$$

where $n_{\alpha}$ is the component of the normal vector on $S_{B}$.

In Case 1, where the field point $P$ is on the control surface

$$
\begin{aligned}
& 2 \pi \sum_{l=-\infty}^{+\infty} \sum_{k=0}^{+\infty} \phi_{k l} \ell_{k}(z) e^{i l \varphi} \\
& =\int_{c}\left[G_{r} \sum_{l=-\infty}^{+\infty} \sum_{k=0}^{+\infty} \psi_{k l} \ell_{k}(\zeta) e^{i l \varphi^{\prime}}-\frac{\partial}{\partial n_{\mathrm{Q}}} G_{r} \sum_{l=-\infty}^{+\infty} \sum_{k=0}^{+\infty} \phi_{k l} \ell_{k}(\zeta) e^{i l \varphi^{\prime}}\right] d s \\
& +\sum_{\alpha=1}^{N_{B}} \int_{B^{\alpha}} G_{r} n_{\alpha} d s-\sum_{\alpha=1}^{N_{B}} \int_{B^{\alpha}} \phi(\mathrm{Q}) \frac{\partial}{\partial n_{\mathrm{Q}}} G_{r} d s \\
& +\frac{\omega^{2}}{g} \sum_{\beta=1}^{N_{F}} \int_{F^{\beta}} G_{r} d s-\sum_{\beta=1}^{N_{F}} \int_{F^{\beta}} \phi(\mathrm{Q}) \frac{\partial}{\partial n_{\mathrm{Q}}} G_{r} d s
\end{aligned}
$$

Application of Eq. (8), one can get 


$$
\begin{aligned}
& 2 \pi \phi_{n m}=\frac{1}{2 \pi} \sum_{l=-\infty}^{+\infty} \sum_{k=0}^{+\infty} \psi_{k l} \int_{0}^{+\infty} \int_{-\pi}^{\pi} \int_{c} G_{r} \ell_{k}(\zeta) e^{i l \varphi^{\prime}} \ell_{n}(z) e^{-i m \varphi} d s d \varphi d z \\
& -\frac{1}{2 \pi} \sum_{l=-\infty}^{+\infty} \sum_{k=0}^{+\infty} \phi_{k l} \int_{0}^{+\infty} \int_{-\pi}^{\pi} \int_{c} \frac{\partial}{\partial n_{\mathrm{Q}}} G_{r} \ell_{k}(\zeta) e^{i l \varphi^{\prime}} \ell_{n}(z) e^{-i m \varphi} d s d \varphi d z \\
& +\frac{1}{2 \pi} \sum_{\alpha=1}^{N_{B}} n_{\alpha} \int_{0}^{+\infty} \int_{-\pi}^{\pi} \int_{B^{\alpha}} G_{r} \ell_{n}(z) e^{-i m \varphi} d s d \varphi d z \\
& -\frac{1}{2 \pi} \sum_{\alpha=1}^{N_{B}} \phi_{\alpha} \int_{0}^{+\infty} \int_{-\pi}^{\pi} \int_{B^{\alpha}} \frac{\partial}{\partial n_{\mathrm{Q}}} G_{r} \ell_{n}(z) e^{-i m \varphi} d s d \varphi d z \\
& -\frac{1}{2 \pi} \sum_{\beta=1}^{N_{F}} \phi_{\beta} \int_{0}^{+\infty} \int_{-\pi}^{\pi} \int_{F^{\beta}}\left[-\frac{\omega^{2}}{g} G_{r}+\frac{\partial}{\partial n_{\mathrm{Q}}} G_{r}\right] \ell_{n}(z) e^{-i m \varphi} d s d \varphi d z
\end{aligned}
$$

Denote

$$
\begin{aligned}
& G_{r, n m, k l}=\frac{1}{2 \pi} \int_{0}^{+\infty} \int_{-\pi}^{\pi} \int_{c} G_{r} \ell_{k}(\zeta) \ell_{n}(z) e^{i l \varphi^{\prime}} e^{-i m \varphi} d s d \varphi d z \\
& H_{r, n m, k l}=\frac{1}{2 \pi} \int_{0}^{+\infty} \int_{-\pi}^{\pi} \int_{c} \frac{\partial}{\partial n_{\mathrm{Q}}} G_{r} \ell_{k}(\zeta) \ell_{n}(z) e^{i l \varphi^{\prime}} e^{-i m \varphi} d s d \varphi d z \\
& G_{B, n m, \alpha}=\frac{1}{2 \pi} \int_{0}^{+\infty} \int_{-\pi}^{\pi} \int_{B^{\alpha}} G_{r} \ell_{n}(z) e^{-i m \varphi} d s d \varphi d z \\
& H_{B, n m, \alpha}=\frac{1}{2 \pi} \int_{0}^{+\infty} \int_{-\pi}^{\pi} \int_{B^{\alpha}} \frac{\partial}{\partial n_{\mathrm{Q}}} G_{r} \ell_{n}(z) e^{-i m \varphi} d s d \varphi d z \\
& G_{F, n m, \beta}=\frac{1}{2 \pi} \int_{0}^{+\infty} \int_{-\pi}^{\pi} \int_{F^{\beta}} G_{r} \ell_{n}(z) e^{-i m \varphi} d s d \varphi d z \\
& H_{F, n m, \beta}=\frac{1}{2 \pi} \int_{0}^{+\infty} \int_{-\pi}^{\pi} \int_{F^{\beta}} \frac{\partial}{\partial n_{\mathrm{Q}}} G_{r} \ell_{n}(z) e^{-i m \varphi} d s d \varphi d z
\end{aligned}
$$

Eq. (49) can then be rewritten as follows

$$
\begin{aligned}
& 2 \pi \phi_{n m}=\sum_{l=-\infty}^{+\infty} \sum_{k=0}^{+\infty} \psi_{k l} G_{r, n m, k l}-\sum_{l=-\infty}^{+\infty} \sum_{k=0}^{+\infty} \phi_{k l} H_{r, n m, k l}+\sum_{\alpha=1}^{N_{B}} n_{\alpha} G_{B, n m, \alpha} \\
& -\sum_{\alpha=1}^{N_{B}} \phi_{\alpha} H_{B, n m, \alpha}-\sum_{\beta=1}^{N_{F}} \phi_{\beta}\left[-\frac{\omega^{2}}{g} G_{F, n m, \beta}+H_{F, n m, \beta}\right]
\end{aligned}
$$

In a similar way with Eq. (21), we can get a matrix form as follows

$$
2 \pi \overrightarrow{\phi_{k l}}=G_{r}^{C, C} \vec{\psi}_{k l}-H_{r}^{C, C} \vec{\phi}_{k l}+G_{r}^{C, B} \vec{n}_{\alpha}-H_{r}^{C, B} \vec{\phi}_{\alpha}-\left(-\frac{\omega^{2}}{g} G_{r}^{C, F}+H_{r}^{C, F}\right) \vec{\phi}_{\beta}
$$

Here $H_{r}^{C, C}, G_{r}^{C, C}, H_{r}^{C, F}, G_{r}^{C, F}, H_{r}^{C, B}$ and $G_{r}^{C, B}$ are matrices composed of $H_{r, n m, k l}$, $G_{r, n m, k l}, H_{F, n m, \beta}, G_{F, n m, \beta}, H_{B, n m, \alpha}$ and $G_{B, n m, \alpha}$, and $\vec{\phi}_{k l}, \vec{\psi}_{k l}$ are vectors consisting of $\phi_{k l}$ and $\psi_{k l}$.

The substitution Eq. (33) into Eq. (52) yields 


$$
\left(2 \pi E_{1}+H_{r}^{C, C}-G_{r}^{C, C} \cdot \overline{D N}\right) \vec{\phi}_{k l}+H_{r}^{C, B} \vec{\phi}_{\alpha}+\left(-\frac{\omega^{2}}{g} G_{r}^{C, F}+H_{r}^{C, F}\right) \vec{\phi}_{\beta}=G_{r}^{C, B} \vec{n}_{\alpha}
$$

In Case 2, when the field point $P$ is on the body surface $S_{B}$ or on the free surface $S_{F}$, the panel including the field point $P$ is numbered by $\gamma$, then Eq. (47) becomes

$$
\begin{aligned}
& 2 \pi \phi_{\gamma}=\sum_{l=-\infty}^{+\infty} \sum_{k=0}^{+\infty} \psi_{k l} \int_{c} G_{r} \ell_{k}(\zeta) e^{i l \varphi^{\prime}} d s-\sum_{l=-\infty}^{+\infty} \sum_{k=0}^{+\infty} \varphi_{k l} \int_{c} \frac{\partial}{\partial n_{\mathrm{Q}}} G_{r} \ell_{k}(\zeta) e^{i l \varphi^{\prime}} d s \\
& +\sum_{\alpha=1}^{N_{B}} n_{\alpha} \int_{B^{\alpha}} G_{r} d s-\sum_{\alpha=1}^{N_{B}} \phi_{\alpha} \int_{B^{\alpha}} \frac{\partial}{\partial n_{\mathrm{Q}}} G_{r} d s-\sum_{\beta=1}^{N_{F}} \phi_{\beta} \int_{F^{\beta}}\left[-\frac{\omega^{2}}{g} G_{r}+\frac{\partial}{\partial n_{\mathrm{Q}}} G_{r}\right] d s
\end{aligned}
$$

Denote

$$
\begin{aligned}
G_{c, \gamma, k l} & =\int_{c} G_{r} \ell_{k}(\zeta) e^{i l \varphi^{\prime}} d s \\
H_{c, \gamma, k l} & =\int_{c} \frac{\partial}{\partial n_{\mathrm{Q}}} G_{r} \ell_{k}(\zeta) e^{i l \varphi^{\prime}} d s \\
G_{B, \gamma, \alpha} & =\int_{B^{\alpha}} G_{r} d s \\
H_{B, \gamma, \alpha} & =\int_{B^{\alpha}} \frac{\partial}{\partial n_{\mathrm{Q}}} G_{r} d s \\
G_{F, \gamma, \beta} & =\int_{F^{\beta}} G_{r} d s \\
H_{F, \gamma, \beta} & =\int_{F^{\beta}} \frac{\partial}{\partial n_{\mathrm{Q}}} G_{r} d s
\end{aligned}
$$

Then Eq. (54) can be rewritten as follows

$$
\begin{aligned}
& 2 \pi \phi_{\gamma}=\sum_{l=-\infty}^{+\infty} \sum_{k=0}^{+\infty} \psi_{k l} G_{\gamma, k l}-\sum_{l=-\infty}^{+\infty} \sum_{k=0}^{+\infty} \phi_{k l} H_{\gamma, k l}+\sum_{\alpha=1}^{N_{B}} n_{\alpha} G_{B, \gamma, \alpha}-\sum_{\alpha=1}^{N_{B}} \phi_{\alpha} H_{B, \gamma, \alpha} \\
& -\sum_{\beta=1}^{N_{F}} \phi_{\beta}\left[-\frac{\omega^{2}}{g} G_{F, \gamma, \beta}+H_{F, \gamma, \beta}\right]
\end{aligned}
$$

In a similar way with Eq. (21), we can get a matrix form as follows

$$
2 \pi E_{3} \vec{\phi}=G_{r}^{B F, C} \vec{\psi}_{k l}-H_{r}^{B F, C} \vec{\phi}_{k l}+G_{r}^{B F, B} \vec{n}_{\alpha}-H_{r}^{B F, B} \vec{\phi}_{\alpha}-\left(-\frac{\omega^{2}}{g} G_{r}^{B F, F}+H_{r}^{B F, F}\right) \vec{\phi}_{\beta}
$$

Here, $G_{r}^{B F, C}, H_{r}^{B F, C}, G_{r}^{B F, B}, H_{r}^{B F, B}, G_{r}^{B F, F}$ and $H_{r}^{B F, F}$ are matrices consisted of $G_{c, \gamma, k l}$, $H_{c, \gamma, k l}, G_{B, \gamma, \alpha}, H_{B, \gamma, \alpha}, G_{F, \gamma, \beta}$ and $H_{F, \gamma, \beta}$ and $\vec{\phi}$ is vector consisted of $\vec{\phi}_{\alpha}$ and $\vec{\phi}_{\beta}, E_{3}$ is a unit matrix with dimensions of $\gamma \times \gamma$,

Substitution of Eq. (33) into Eq. (57) yields

$$
\left(H_{r}^{B F, C}-G_{r}^{B F, C} \overline{D N}\right) \vec{\phi}_{k l}+\left(H_{r}^{B F, B},-\frac{\omega^{2}}{g} G_{r}^{B F, F}+H_{r}^{B F, F}\right) \vec{\phi}+2 \pi E_{3} \vec{\phi}=G_{r}^{B F, B} \vec{n}_{\alpha}
$$


Then the equations for the entire domain can be obtained by combining Eq. (53) and (58)

$$
\left[\begin{array}{c}
2 \pi E_{1}+H_{r}^{C, C}-G_{r}^{C, C} \cdot \overline{D N}, \\
H_{r}^{B F, C}-G_{r}^{B F, C} \overline{D N} \quad,\left(H_{r}^{C, B},-\frac{\omega^{2}}{g} G_{r}^{C, F}+H_{r}^{C, F}\right) \\
\left.H_{r}^{B F, B},-\frac{\omega^{2}}{g} G_{r}^{B F, F}+H_{r}^{B F, F}\right)+2 \pi E_{3}
\end{array}\right]\left[\begin{array}{l}
\vec{\phi}_{k l} \\
\vec{\phi}
\end{array}\right]=\left[\begin{array}{l}
G_{r}^{C, B} \\
G_{r}^{B F, B}
\end{array}\right] \vec{n}_{\alpha}
$$

Once Eq. (59) is solved, one can obtain the velocity potential on the panels and the series expansion coefficients of the velocity potential on the control surface. The method for evaluating the coefficients we have defined for the multi-fold integrals is given in the appendices. From the expression of the multi-fold integrals, the Chebyshev expansion is used to approximate the integrations and improve the efficiency.

\subsection{The solution of added mass and damping coefficient}

The added mass and damping coefficient can be solved as follows

$$
a_{i j}+\frac{i b_{i j}}{\omega}=\rho \iint_{S_{b}} \phi_{j} \frac{\partial \phi_{i}}{\partial n} d s
$$

where, $a_{i j}$ is added mass, $b_{i j}$ is the damping coefficient, as the body surface is discretized into panels, Eq. (60) can be rewritten as

$$
a_{i j}+\frac{i b_{i j}}{\omega}=\rho \iint_{S_{b}} \phi_{j} \frac{\partial \phi_{i}}{\partial n} d s=\rho \sum_{\alpha=1}^{N_{\alpha}} \phi_{j}^{\alpha} n_{i}^{\alpha} \quad(i, j=1,2, \cdots, 6)
$$

Then added mass and damping coefficients can be obtained by substituting the velocity potential on the body surface into Eq. (61).

\section{Results and analysis}

\subsection{Results for the diffraction potential}

Numerical solution has been computed with the order of Laguerre function from 0 to 10 and the order of Fourier series from -10 to 10 , the radius of the cylinder is $3.0 \mathrm{~m}$, a fixed point $P(3.01 .51 .0)$ is selected expressed in cylindrical coordinates on the circular cylinder, the diffraction potentials at the fixed point varying with wavenumber are shown in Figure 3, among them, method 1 refers to the original solution method of the exterior domain in subsection 3.2, Method 2 refers to the extended boundary integral equation method in subsection 3.3. 


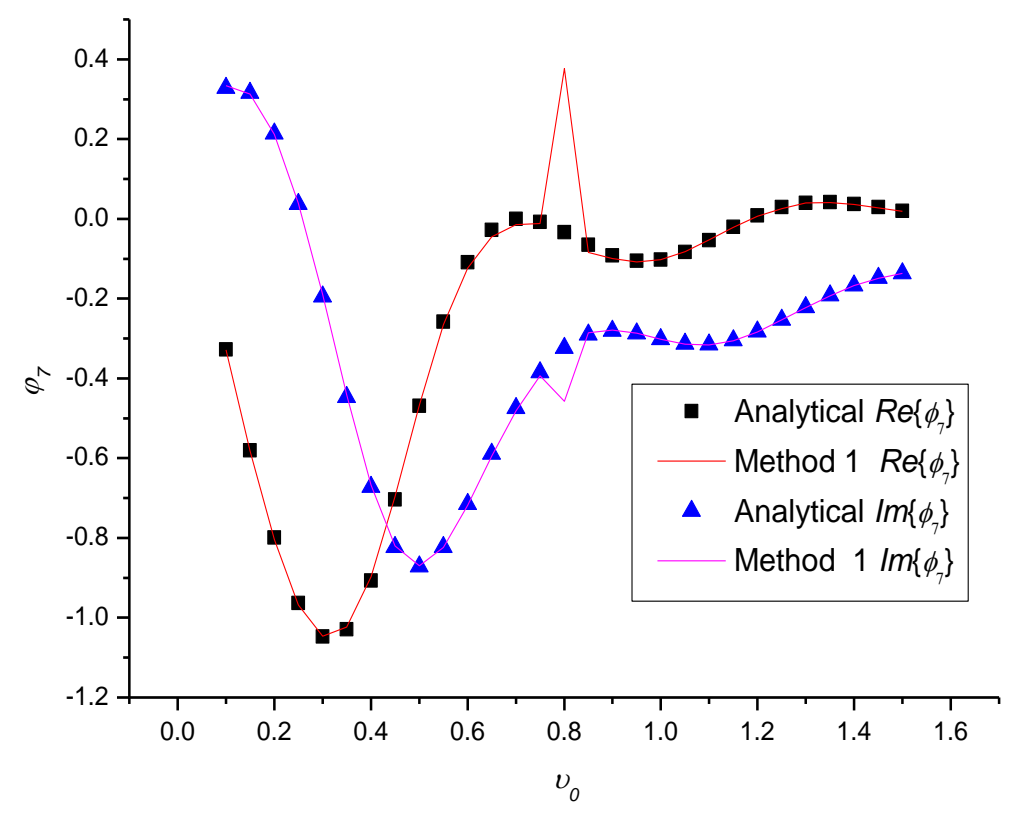

Fig. 3 The comparison results of diffraction potentials

In Figure 3, the numerical solution agrees well with analytical solution for wavenumber varying from 0.1 to 1.6 except for $v_{0}$ is 0.8 .

As we can see, numerical solution has a fluctuation when wavenumber is 0.8 , and this wave number is regarded as irregular frequency. Different points on the circular cylinder are chosen with $h=3.0$ and $z=1.0$. The diffraction potentials at the irregular frequency varying with different circumferential locations of the points are shown in Figure 4

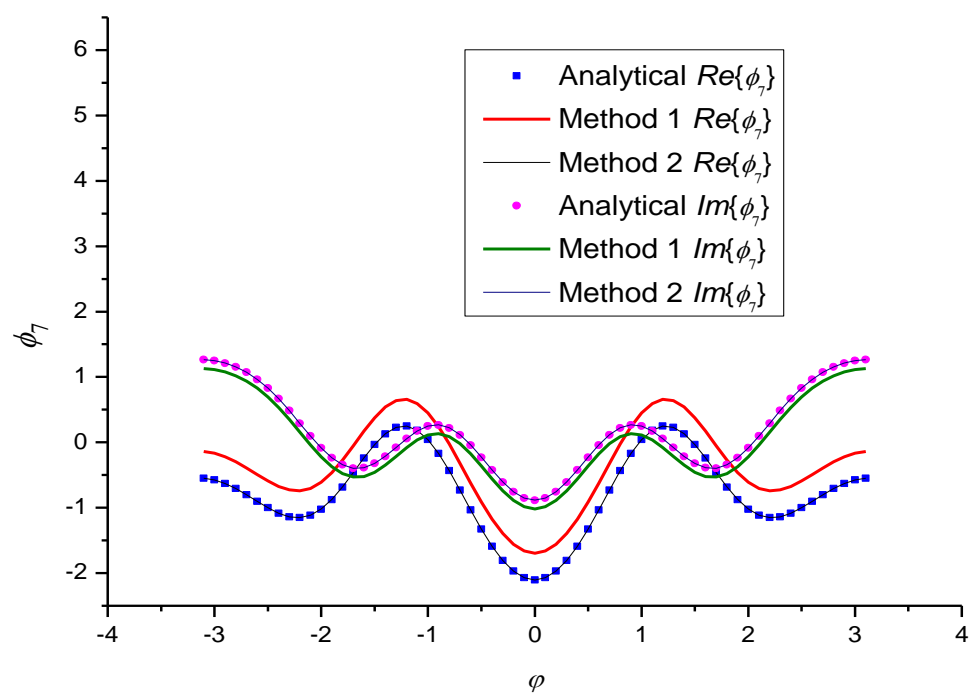

Fig. 4 The comparison results of diffraction potentials at irregular frequency

From Figure 4, we can see that the numerical solution obtained from extended boundary integral equations in method 2 is in good agreement with analytical solution, which illustrates the relationship between the velocity potential and its normal derivative on control surface is right and extended boundary integral equation method is capable of removing the irregular frequency. 


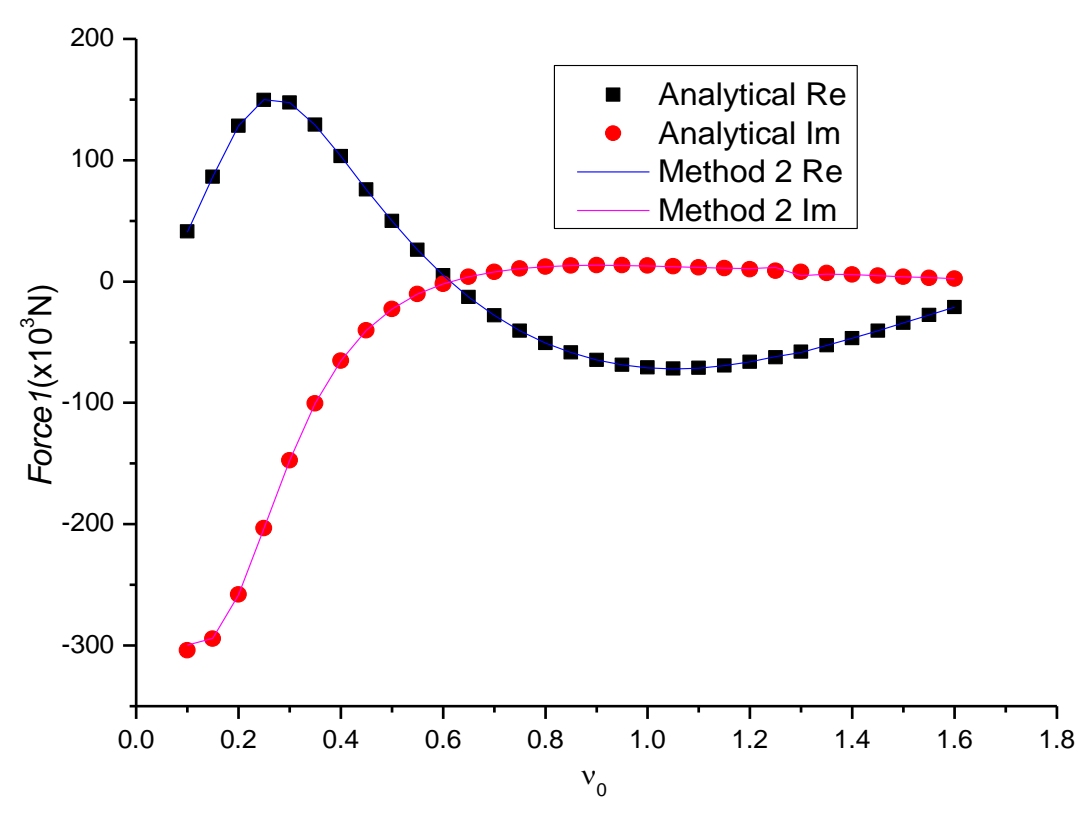

Fig. 5 The comparison results of diffraction force

Diffraction force in the direction of $x$ axis is given in Figure 5, and the numerical solution shows a satisfied accuracy.

\subsection{The results of added mass and damping coefficients of a hemisphere}

A hemisphere is chosen as the example for calculation, the numerical solutions about the added mass and damping coefficient are compared with the analytical solutions given by Hulme[8]. For the convenience, we have defined the following conditions shown in table 1.

Table 1 list of conditions

\begin{tabular}{|l|c|c|c|c|c|c|}
\hline \multirow{2}{*}{\multicolumn{1}{|c|}{ Parameter's name }} & \multirow{2}{*}{ Notation } & \multicolumn{5}{|c|}{ Condition } \\
\cline { 3 - 7 } & & 1 & 2 & 3 & 4 & 5 \\
\hline Radius of the hemisphere & $\mathrm{r}$ & $2 \mathrm{~m}$ & $2 \mathrm{~m}$ & $2 \mathrm{~m}$ & $2 \mathrm{~m}$ & $2 \mathrm{~m}$ \\
\hline Radius of the control surface & $\mathrm{R}$ & $3 \mathrm{~m}$ & $3 \mathrm{~m}$ & $3 \mathrm{~m}$ & $4 \mathrm{~m}$ & $6 \mathrm{~m}$ \\
\hline Number of panels of hemisphere & $N_{B}$ & 300 & 300 & 300 & 300 & 300 \\
\hline Number of panels on free surface & $N_{F}$ & 1900 & 1900 & 1900 & 2500 & 3190 \\
\hline Order of Laguerre function & $\mathrm{N}$ & 5 & 10 & 20 & 20 & 20 \\
\hline Order of Fourier series & $\mathrm{M}$ & 5 & 10 & 20 & 20 & 20 \\
\hline
\end{tabular}

The convergence test associated with the order of Fourier-Laguerre series has been made through condition 1,2 and 3 . The results are shown in the following figures, among them, $L$ is the characteristic length which is equal to $r$ the radius of hemisphere. 


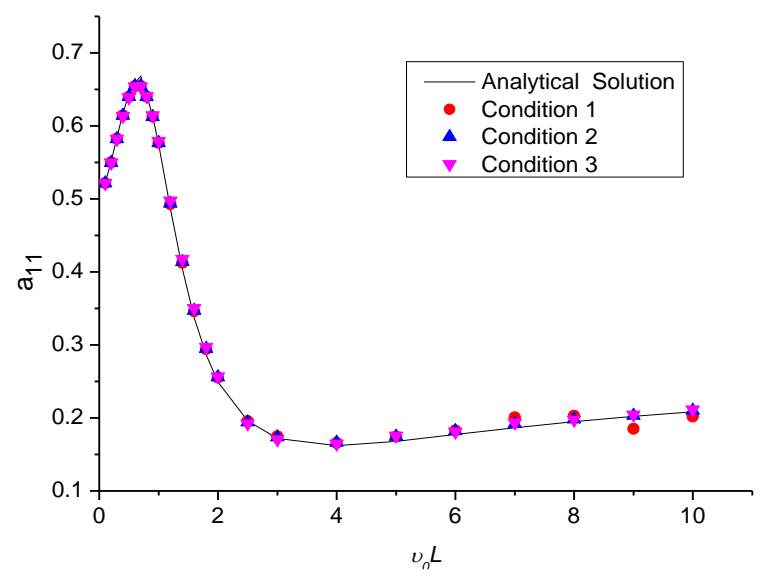

Fig. 6 Surge added mass coefficients with different order of Fourier-Laguerre series

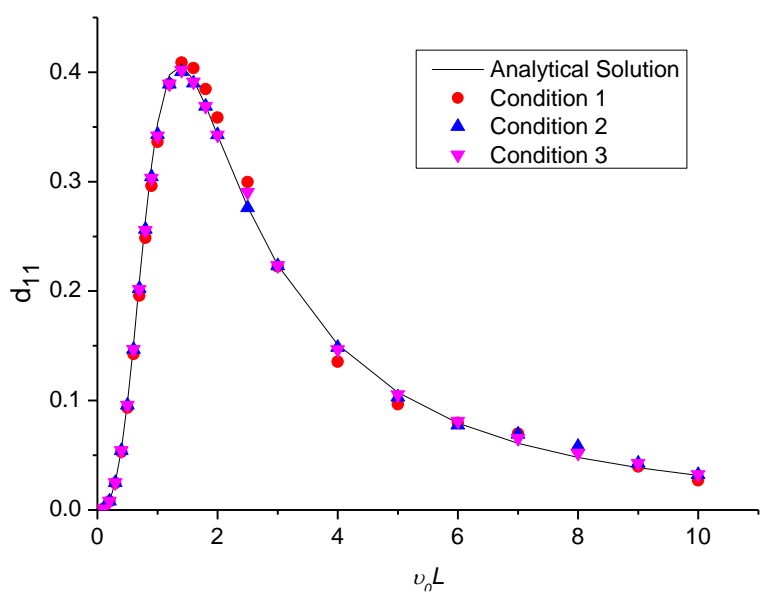

Fig. 8 Surge damping coefficients with different order of Fourier-Laguerre series

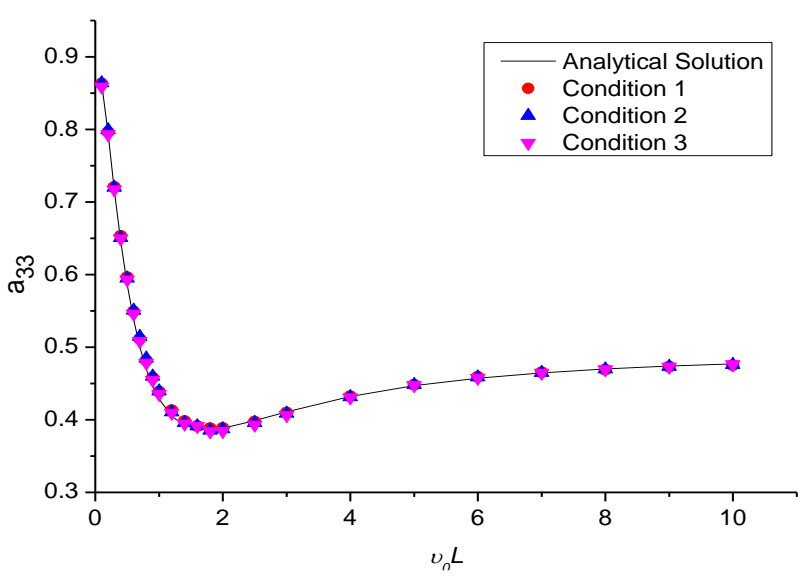

Fig. 7 Heave added mass coefficients with different order of Fourier-Laguerre series

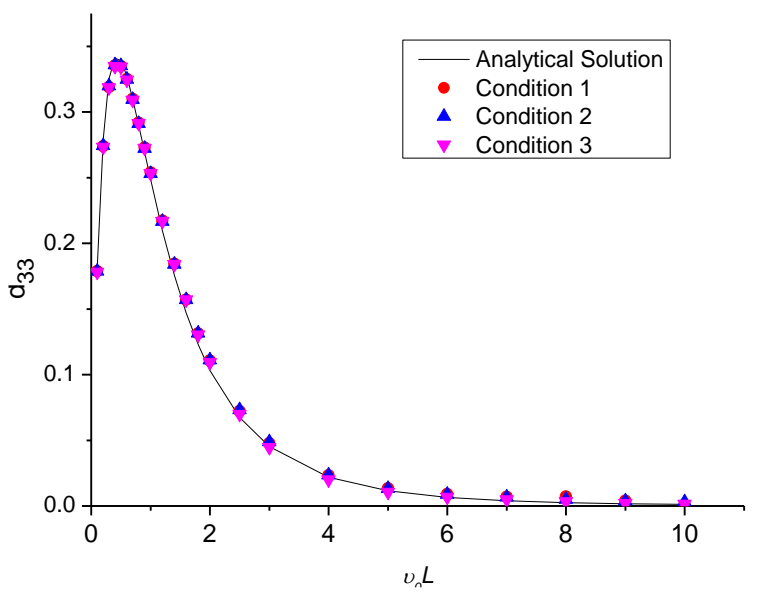

Fig. 9 Heave damping coefficients with different order of Fourier-Laguerre series

From the results, we can see that the numerical results are in good agreement with the analytical solutions, in Figure 6 and Figure 8. The results of surge motion seem sensitive to the order of Fourier-Laguerre series at a high wave frequency, and the discrepancy between analytical solutions and numerical results in condition 1 are larger than that in condition 2 and condition 3. From Figure 7 and Figure 9, the results of heave added mass and damping coefficients have shown a good precision in condition 1 . In conclusion, the numerical results show a good convergence with the increase of the order of Fourier-Laguerre series.

At the same time, the influence of different control surface radius to numerical results has also been studied through condition 3,4 and 5. The results are shown in the following figures. 


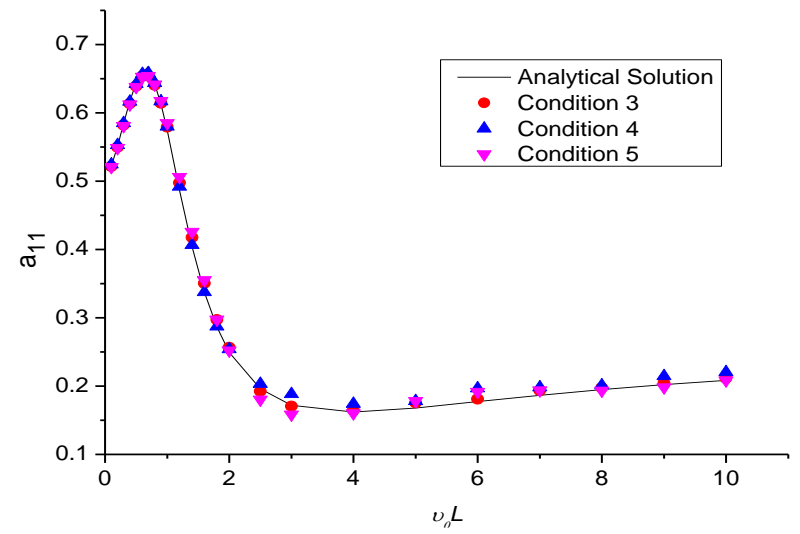

Fig. 10 Surge added mass coefficients for different control surface radius

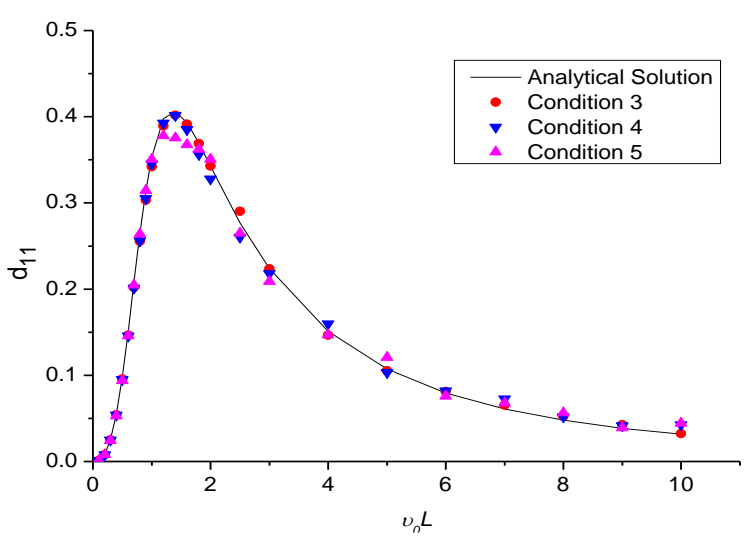

Fig. 12 Surge damping coefficients for different control surface radius

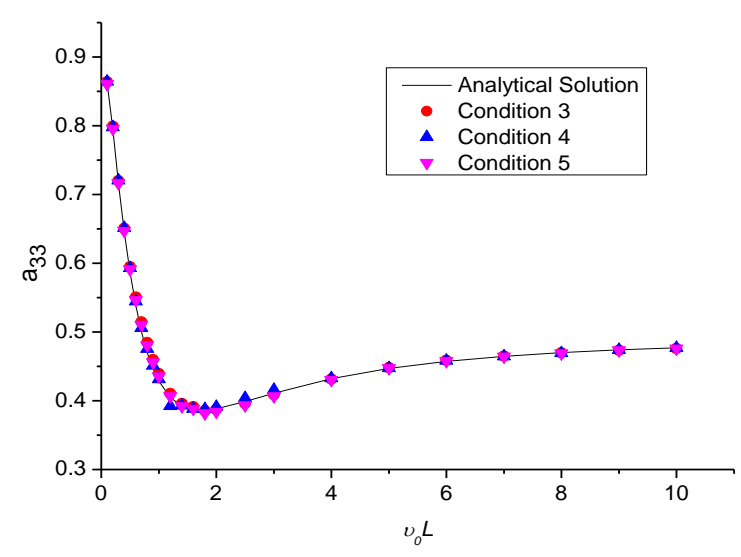

Fig. 11 Heave added mass coefficients for different control surface radius

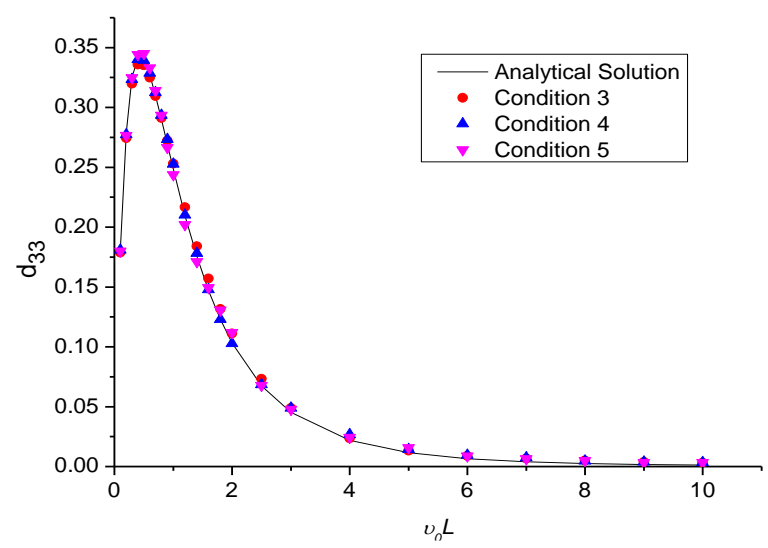

Fig. 13 Heave damping coefficients for different control surface radius

From the results we can see, added mass and damping coefficients of surge motion are more sensitive to control surface radius than that of heave motion. In Figure 11 and Figure 13, the numerical results show a good agreement with the analytical solution in different conditions. In Figure 10 and Figure 12, the influence of the control surface radius to the numerical result is small at a low wave frequency, and with the increase of the wavenumber, the numerical results in condition 3 are more accurate than the other conditions overall. The main reason is more panels are needed on the free surface with the increase of the control surface radius. Consequently, considering the chosen of control surface radius, we prefer to a smaller one.

\section{Conclusion}

The Rankine-Kelvin hybrid method has been applied to solve zero speed seakeeping problems successfully. From this paper, we can draw the following conclusions

1. The application of Kelvin source in the external domain will result in irregular frequency, and an extended boundary integral equation method has been used to eliminate the irregular frequencies.

2. The result is convergent with the increase of the order of Fourier-Laguerre series, and a better accuracy can be achieved when a smaller radius of the control surface is chosen with the same order of Fourier-Laguerre series.

3. All the work in this paper has laid the foundation for solving the forward speed hydrodynamic problem. 


\section{ACKNOWLEDGMENTS}

This research is funded by China MOST 973 plan (2011CB013703). The discussions with Dr. Liang Hui and Chen Xiao-Bo of Bureau Veritas are appreciated.

\section{REFERENCES}

[1] Chen X.B., Wu G.X. (2001). On singular and highly oscillatory properties of the Green function for ship motions. Journal of Fluid Mechanics, Vol 445, 77-91. http://dx.doi.org/10.1017/S0022112001005481.

[2] Chen X. B., Diebold L., Doutreleau Y. (2001). New Green-function method to predict wave-induced ship motions and loads. In Twenty-Third Symposium on Naval Hydrodynamics. Val de Reuil, France, September 17-22.

[3] Ten I., Chen X.B. (2010). A coupled Rankine-Green function method applied to the forward-speed seakeeping problem. 25th IWWWFB, Harbin, China, May 9-12.

[4] Liang H., Chen X.B. (2016). A multi-domain method for the computation of wave loads. 31th IWWWFB, Plymouth, MI, USA, April 3-6.

[5] Lv M.D., Li H., Ren H.L., Chen X.B. (2015). Expansion by Laguerre Function for Wave Diffraction around an Infinite Cylinder. In Proceedings of Computational Mathematics and Applications Conference, 75-80. http://dx.doi.org/10.4236/jamp.2015.31010.

[6] Wehausen J.V., Laitone E.V. (1960). Surface Waves, in Flügge S., Truesdell C., eds., Encyclopaedia of Physics (Springer Verlag), Vol 9: 446-778. http://dx.doi.org/10.1007/978-3-642-45944-3 6.

[7] Zhu X. (1994). Irregular frequency removal from the boundary integral equation for the wave-body problem (Doctoral dissertation), Massachusetts Institute of Technology.

[8] Hulme A. (1982). Wave forces acting on a floating hemisphere undergoing forced periodic oscillations. Journal of Fluid Mechanics, Vol 121(1), 443-463. http://dx.doi.org/10.1017/S0022112082001980.

[9] Chen X.B. (2014). Personal notes on Fourier-Laguerre expansion on an infinite vertical cylinder. 


\section{APPENDIX 1} notes [9].

In this part, some integrals about Laguerre function will be calculated following his

1 The calculation about the integration of Laguerre function

1.1

$$
\begin{aligned}
& \int_{z}^{+\infty} e^{-v \zeta} L_{k}(\zeta) d \zeta=\int_{z}^{+\infty} e^{-v \zeta}\left[L_{k}^{\prime}(\zeta)-L_{k+1}^{\prime}(\zeta)\right] d \zeta \\
& =-e^{-v z} L_{k}(z)+e^{-v z} L_{k+1}(z)-\int_{z}^{+\infty} v e^{-v \zeta} L_{k+1}(\zeta) d \zeta+\int_{z}^{+\infty} v e^{-v \zeta} L_{k}(\zeta) d \zeta
\end{aligned}
$$

Eq. (1.1) can be transformed into recursion Eq. (1.2)

$$
\int_{z}^{+\infty} e^{-v \zeta} L_{k+1}(\zeta) d \zeta=\frac{1}{v} e^{-v z}\left[L_{k+1}(z)-L_{k}(z)\right]-\frac{1-v}{v} \int_{z}^{+\infty} e^{-v \zeta} L_{k}(\zeta) d \zeta \quad(v \neq 0)
$$

Change the order of Laguerre polynomial from $k+1$ into $k$ and reuse Eq. (1.2), we can get the following result

$$
\begin{aligned}
& \int_{z}^{+\infty} e^{-v \zeta} L_{k}(\zeta) d \zeta=\frac{1}{v} e^{-v z}\left[L_{k}(z)-L_{k-1}(z)\right]-\frac{1-v}{v} \int_{z}^{+\infty} e^{-v \zeta} L_{k-1}(\zeta) d \zeta \\
& =\sum_{i=0}^{k-1} \frac{1}{v} e^{-v z}\left(-\frac{1-v}{v}\right)^{i}\left[L_{k-i}(z)-L_{k-i-1}(z)\right]+\left(-\frac{1-v}{v}\right)^{k}\left(\frac{1}{v} e^{-v z}\right)
\end{aligned}
$$

Finally, the integration about Laguerre function can be solved referring to Eq. (1.3)

$$
\begin{aligned}
& \int_{z}^{+\infty} e^{-v \zeta} \ell_{k}(\zeta) d \zeta=\int_{z}^{+\infty} e^{-\left(v+\frac{1}{2}\right) \zeta} L_{k}(\zeta) d \zeta \\
& =\sum_{i=0}^{k-1} \frac{2}{2 v+1} e^{-\left(v+\frac{1}{2}\right) z}\left(-\frac{1-2 v}{2 v+1}\right)^{i}\left[L_{k-i}(z)-L_{k-i-1}(z)\right]+\left(-\frac{1-2 v}{2 v+1}\right)^{k}\left(\frac{2}{2 v+1} e^{-\left(v+\frac{1}{2}\right) z}\right) \\
& \quad\left(v \neq-\frac{1}{2}\right)
\end{aligned}
$$

1.2

$$
\begin{aligned}
& \int_{0}^{z} e^{\nu \zeta} L_{k}(\zeta) d \zeta=\int_{0}^{z} e^{v \zeta}\left[L_{k}^{\prime}(\zeta)-L_{k+1}^{\prime}(\zeta)\right] d \zeta \\
& =e^{\nu z} L_{k}(z)-1-e^{\nu z} L_{k+1}(z)+1-\int_{0}^{z} v e^{\nu \zeta} L_{k}(\zeta) d \zeta+\int_{0}^{z} v e^{v \zeta} L_{k+1}(\zeta) d \zeta
\end{aligned}
$$

Eq. (1.5) can be transformed into recursion Eq. (1.6)

$$
\begin{aligned}
& \int_{0}^{z} e^{v \zeta} L_{k}(\zeta) d \zeta=\frac{1}{v} e^{v z} L_{k}(z)-\frac{1}{v} e^{v z} L_{k-1}(z)+\frac{v+1}{v} \int_{0}^{z} e^{v \zeta} L_{k-1}(\zeta) d \zeta \\
& =\sum_{i=0}^{k-1} \frac{1}{v} e^{v z}\left(\frac{1+v}{v}\right)^{i}\left[L_{k-i}(z)-L_{k-i-1}(z)\right]+\left(\frac{1+v}{v}\right)^{k} \frac{1}{v}\left(e^{v z}-1\right)
\end{aligned}
$$

Finally, the integration about Laguerre function can be solved referring to Eq. (1.6) 


$$
\begin{aligned}
& \int_{0}^{z} e^{v \zeta} \ell_{k}(\zeta) d \zeta=\int_{0}^{z} e^{\left(v-\frac{1}{2}\right) \zeta} L_{k}(\zeta) d \zeta \\
& =\sum_{i=0}^{k-1} \frac{2}{2 v-1} e^{\left(v-\frac{1}{2}\right) z}\left(\frac{2 v+1}{2 v-1}\right)^{i}\left[L_{k-i}(z)-L_{k-i-1}(z)\right]+\left(\frac{2 v+1}{2 v-1}\right)^{k}\left(\frac{2}{2 v-1}\left(e^{\left(v-\frac{1}{2}\right) z}-1\right)\right) \\
& \quad\left(v \neq \frac{1}{2}\right)
\end{aligned}
$$

When $v=\frac{1}{2}$

$$
\int_{0}^{z} e^{\nu \zeta} \ell_{k}(\zeta) d \zeta=\int_{0}^{z} L_{k}(\zeta) d \zeta=L_{k}(z)-L_{k+1}(z)
$$

When the integration is computed near $v=\frac{1}{2}$, we have done the following approximation

$$
e^{(v-1 / 2) \zeta}=\sum_{m=0}^{\infty} \frac{1}{m !}\left(v-\frac{1}{2}\right)^{m} \zeta^{m}
$$

Then we have

$$
\begin{aligned}
\int_{0}^{z} e^{(v-1 / 2) \zeta} L_{n}(\zeta) d \zeta & =\int_{0}^{z}\left[\sum_{m=0}^{\infty} \frac{1}{m !}\left(v-\frac{1}{2}\right)^{m} \zeta^{m}\right] L_{n}(\zeta) d \zeta \\
& =\sum_{m=0}^{\infty} \sum_{k=0}^{n} \frac{(-1)^{k} n !}{m !(k !)^{2}(n-k) !} \frac{1}{m+k+1}\left[\left(v-\frac{1}{2}\right) z\right]^{m} z^{k+1}
\end{aligned}
$$

$$
1.3 \int_{0}^{+\infty} e^{v z} \ell_{n}(z) d z \int_{z}^{+\infty} e^{-v \zeta} \ell_{k}(\zeta) d \zeta
$$

We use the result of Eq. (1.4), then

$$
\begin{aligned}
& \int_{0}^{+\infty} e^{v z} \ell_{n}(z) d z \int_{z}^{+\infty} e^{-v \zeta} \ell_{k}(\zeta) d \zeta \\
& =\sum_{i=0}^{k-1} \frac{2}{2 v+1}\left(-\frac{1-2 v}{2 v+1}\right)^{i} \int_{0}^{+\infty} e^{-z} L_{n}(z)\left[L_{k-i}(z)-L_{k-i-1}(z)\right] d z+\left(-\frac{1-2 v}{2 v+1}\right)^{k} \frac{2}{2 v+1} \int_{0}^{+\infty} e^{-z} L_{n}(z) d z
\end{aligned}
$$

Referring to Eq. (2), the analytical solution of Eq. (1.11) can be obtained as follow

$$
\begin{array}{ll}
\int_{0}^{+\infty} e^{v z} \ell_{n}(z) d z \int_{z}^{+\infty} e^{-v \zeta} \ell_{k}(\zeta) d \zeta \\
=\frac{-4(2 v-1)^{k-n-1}}{(2 v+1)^{k-n+1}} & (0 \leq n \leq k-1) \\
=\frac{2}{2 v+1} & (n=k) \\
=0 & (n>k)
\end{array}
$$




$$
1.4 \int_{0}^{+\infty} e^{-v z} \ell_{n}(z) d z \int_{0}^{z} e^{v \zeta} \ell_{k}(\zeta) d \zeta
$$

Similar to Eq. (1.11), we can get the following result

$$
\begin{array}{ll}
\int_{0}^{+\infty} e^{-v z} \ell_{n}(z) d z \int_{0}^{z} e^{v \zeta} \ell_{k}(\zeta) d \zeta & \\
=0 & (0 \leq n \leq k-1) \\
=\frac{2}{2 v+1} & (n=k) \\
=\frac{-4(2 v+1)^{k-n-1}}{(2 v-1)^{k-n+1}} & (n>k)
\end{array}
$$

\section{APPENDIX 2} system.

This part introduces the expression of Green function in the cylindrical coordinate

Kelvin source can be written as Eq. (2.1)

$$
\begin{aligned}
& G(P, \mathrm{Q})=G_{r}+G_{r^{\prime}}+G_{w}+G_{c} \\
& =\frac{1}{r_{P \mathrm{Q}}}+\frac{1}{r_{P \overline{\mathrm{Q}}}}+2 v_{0} P \cdot V \cdot \int_{0}^{+\infty} \frac{1}{v-v_{0}} e^{-v(z+\zeta)} J_{0}(v R) d v+i 2 \pi v_{0} e^{-v_{0}(z+\zeta)} J_{0}\left(v_{0} R\right)
\end{aligned}
$$

among them,

$G_{r}=\frac{1}{r_{P Q}}, G_{r^{\prime}}=\frac{1}{r_{P \bar{Q}}}, G_{w}=2 v_{0} P . V \cdot \int_{0}^{+\infty} \frac{1}{v-v_{0}} e^{-v(z+\zeta)} J_{0}(v R) d v, G_{c}=i 2 \pi v_{0} e^{-v_{0}(z+\zeta)} J_{0}\left(v_{0} R\right)$.

$G_{r}$ is the Rankine source which is associated with the distance between the field point $P(x, y, z)$ and the source point $\mathrm{Q}(\xi, \eta, \zeta)$ :

$$
G_{r}=\frac{1}{r_{P Q}}=1 / \sqrt{(x-\xi)^{2}+(y-\eta)^{2}+(z-\zeta)^{2}}=1 / \sqrt{R^{2}+Z^{2}}
$$

Among them $R=\sqrt{(x-\xi)^{2}+(y-\eta)^{2}}, Z=|z-\zeta|$

Eq. (2.2) can be rewritten as

$$
1 / \sqrt{R^{2}+Z^{2}}=\int_{0}^{\infty} e^{-v Z} J_{0}(v R) d v
$$

In the cylindrical coordinate system, the field point $P$ and the source point $\mathrm{Q}$ can be written as $P(h, \varphi, z), \mathrm{Q}\left(h^{\prime}, \varphi^{\prime}, \zeta\right)$

Using the identity

$$
J_{0}(v R)=\sum_{p=-\infty}^{+\infty} e^{i p\left(\varphi-\varphi^{\prime}\right)} J_{p}(v h) J_{p}\left(v h^{\prime}\right)
$$

We can get 


$$
G_{r}=\sum_{p=-\infty}^{+\infty} e^{i p\left(\varphi-\varphi^{\prime}\right)} \int_{0}^{\infty} e^{-v|z-\zeta|} J_{p}(v h) J_{p}\left(v h^{\prime}\right) d v
$$

In a similar way

$$
\begin{aligned}
& G_{r^{\prime}}=\sum_{p=-\infty}^{+\infty} e^{i p\left(\varphi-\varphi^{\prime}\right)} \int_{0}^{\infty} e^{-v(z+\zeta)} J_{p}(v h) J_{p}\left(v h^{\prime}\right) d v \\
& G_{w}=2 v_{0} \sum_{p=-\infty}^{+\infty} e^{i p\left(\varphi-\varphi^{\prime}\right)} P \cdot V \cdot \int_{0}^{+\infty} \frac{1}{v-v_{0}} e^{-v(z+\zeta)} J_{p}(v h) J_{p}\left(v h^{\prime}\right) d v \\
& G_{c}=i 2 \pi v_{0} e^{-v_{0}(z+\zeta)} \sum_{p=-\infty}^{+\infty} e^{i p\left(\varphi-\varphi^{\prime}\right)} J_{p}\left(v_{0} h\right) J_{p}\left(v_{0} h^{\prime}\right)
\end{aligned}
$$

\section{APPENDIX 3}

The computational methods of the integrals defined in the external domain.

$G_{n m, k l}$ can be divided into the following four parts

$$
\begin{aligned}
& G_{n m, k l 1}=\frac{1}{2 \pi} \int_{0}^{+\infty} \int_{-\pi}^{\pi} \int_{c} G_{r} \ell_{k}(\zeta) \ell_{n}(z) e^{i l \varphi^{\prime}} e^{-i m \varphi} d s d \varphi d z \\
& G_{n m, k l 2}=\frac{1}{2 \pi} \int_{0}^{+\infty} \int_{-\pi}^{\pi} \int_{c} G_{r^{\prime}} \ell_{k}(\zeta) \ell_{n}(z) e^{i l \varphi^{\prime}} e^{-i m \varphi} d s d \varphi d z \\
& G_{n m, k l 3}=\frac{1}{2 \pi} \int_{0}^{+\infty} \int_{-\pi}^{\pi} \int_{c} G_{w} \ell_{k}(\zeta) \ell_{n}(z) e^{i l \varphi^{\prime}} e^{-i m \varphi} d s d \varphi d z \\
& G_{n m, k l 4}=\frac{1}{2 \pi} \int_{0}^{+\infty} \int_{-\pi}^{\pi} \int_{c} G_{c} \ell_{k}(\zeta) \ell_{n}(z) e^{i l \varphi^{\prime}} e^{-i m \varphi} d s d \varphi d z
\end{aligned}
$$

Then we can calculate the four parts separately with the formulas mentioned in appendixes 1 and 2. Substitute Eq. (2.5) into Eq. (3.1), use the orthogonally of Fourier series, we can get Eq. (3.5)

$$
\begin{aligned}
G_{n m, k l 1} & =\frac{1}{2 \pi} \int_{0}^{+\infty} \int_{-\pi}^{\pi} \int_{c} G_{r} \ell_{k}(\zeta) \ell_{n}(z) e^{i l \varphi^{\prime}} e^{-i m \varphi} d s d \varphi d z \\
& =2 \pi h^{\prime} \sum_{p=-\infty}^{+\infty} \int_{0}^{+\infty} J_{p}(v h) J_{p}\left(v h^{\prime}\right) d v \int_{0}^{+\infty} e^{v z} \ell_{n}(z) d z \int_{z}^{+\infty} e^{-v \zeta} \ell_{k}(\zeta) d \zeta \cdot \delta_{p m} \delta_{p l} \\
& +2 \pi h^{\prime} \sum_{p=-\infty}^{+\infty} \int_{0}^{+\infty} J_{p}(v h) J_{p}\left(v h^{\prime}\right) d v \int_{0}^{+\infty} e^{-v z} \ell_{n}(z) d z \int_{0}^{z} e^{v \zeta} \ell_{k}(\zeta) d \zeta \cdot \delta_{p m} \delta_{p l}
\end{aligned}
$$

Substitute Eq. (1.12) and (1.13) into Eq. (3.5), we can obtain the following results When $n=k$

$$
G_{n m, k l 1}=4 \pi h^{\prime} \sum_{p=-\infty}^{+\infty} \int_{0}^{+\infty} J_{p}(v h) J_{p}\left(v h^{\prime}\right) \frac{2}{2 v+1} d v \cdot \delta_{p m} \cdot \delta_{p l}
$$


Otherwise

$$
G_{n m, k l 1}=2 \pi h^{\prime} \sum_{p=-\infty}^{+\infty} \int_{0}^{+\infty} J_{p}(v h) J_{p}\left(v h^{\prime}\right) \frac{-4}{(2 v-1)(2 v+1)}\left(\frac{2 v-1}{2 v+1}\right)^{|k-n|} d v \cdot \delta_{p m} \cdot \delta_{p l}
$$

In a similar way

$$
\begin{aligned}
& G_{n m, k l 2}=8 \pi h^{\prime} \sum_{p=-\infty}^{\infty} \int_{0}^{+\infty} J_{p}(v h) J_{p}\left(v h^{\prime}\right) \frac{(2 v-1)^{n+k}}{(2 v+1)^{n+k+2}} d v \cdot \delta_{p m} \delta_{p l} \\
& G_{n m, k l 3}=16 \pi h^{\prime} v_{0} \sum_{p=-\infty}^{+\infty} \delta_{p l} \delta_{p m} \cdot p \cdot v \cdot \int_{0}^{+\infty} \frac{1}{v-v_{0}} J_{p}^{2}(v h) \frac{(2 v-1)^{n+k}}{(2 v+1)^{n+k+2}} d v
\end{aligned}
$$

To make the calculation of numerical method accurate and efficient, we can deal with Eq. (3.9) as follows

$$
\begin{aligned}
& G_{n m, k l 3}=16 \pi h^{\prime} v_{0} \sum_{p=-\infty}^{+\infty} \delta_{p l} \delta_{p m} \cdot p \cdot v \cdot \int_{0}^{+\infty} \frac{1}{v-v_{0}} J_{p}^{2}(v h) \frac{(2 v-1)^{n+k}}{(2 v+1)^{n+k+2}} d v \\
& \pm 16 \pi h^{\prime} v_{0} \sum_{p=-\infty}^{+\infty} \delta_{p l} \delta_{p m} \cdot p \cdot v \cdot \int_{0}^{2 v_{0}} \frac{1}{v-v_{0}} J_{p}^{2}\left(v_{0} h\right) \frac{\left(2 v_{0}-1\right)^{n+k}}{\left(2 v_{0}+1\right)^{n+k+2}} d v \\
& =16 \pi h^{\prime} v_{0} \sum_{p=-\infty}^{+\infty} \delta_{p l} \delta_{p m} \cdot p \cdot v \cdot \int_{0}^{2 v_{0}}\left[\frac{1}{v-v_{0}} J_{p}^{2}(v h) \frac{(2 v-1)^{n+k}}{(2 v+1)^{n+k+2}}-\frac{1}{v-v_{0}} J_{p}^{2}\left(v_{0} h\right) \frac{\left(2 v_{0}-1\right)^{n+k}}{\left(2 v_{0}+1\right)^{n+k+2}}\right] d v \\
& +16 \pi h^{\prime} v_{0} \sum_{p=-\infty}^{+\infty} \delta_{p l} \delta_{p m} \cdot \int_{2 v_{0}}^{+\infty} \frac{1}{v-v_{0}} J_{p}^{2}(v h) \frac{(2 v-1)^{n+k}}{(2 v+1)^{n+k+2}} d v
\end{aligned}
$$

Substitute Eq. (2.8) into (3.4), we can get

$$
\begin{aligned}
G_{n m k l 4} & =\frac{1}{2 \pi} \int_{0}^{+\infty} \int_{-\pi}^{\pi} \int_{c}\left\{i 2 \pi v_{0} e^{-v_{0}(z+\zeta)} J_{0}\left(v_{0} R\right)\right\} \ell_{k}(\zeta) \ell_{n}(z) e^{i l \varphi^{\prime}} e^{-i m \varphi} d s d \varphi d z \\
& =16 \pi^{2} v_{0} h^{\prime} i \sum_{p=-\infty}^{+\infty} \delta_{p m} \delta_{p l} \cdot J_{p}\left(v_{0} h\right) J_{p}\left(v_{0} h^{\prime}\right) \frac{\left(2 v_{0}-1\right)^{n+k}}{\left(2 v_{0}+1\right)^{n+k+2}}
\end{aligned}
$$

The normal derivative of Kelvin source on the control surface can be written as

$$
\frac{\partial}{\partial n_{\mathrm{Q}}} G=\frac{\partial}{\partial h^{\prime}} G=\frac{\partial}{\partial h^{\prime}}\left(G_{r}+G_{r^{\prime}}+G_{w}+G_{c}\right)
$$

Then

$$
\frac{\partial}{\partial n_{\mathrm{Q}}} G_{r}=\sum_{p=-\infty}^{+\infty} e^{i p\left(\psi-\psi^{\prime}\right)} \int_{0}^{+\infty} v e^{-\kappa|\zeta-z|} J_{p}(v h) J_{p}^{\prime}\left(v h^{\prime}\right) d v
$$




$$
\begin{aligned}
& \frac{\partial}{\partial n_{\mathrm{Q}}} G_{r^{\prime}}=\sum_{p=-\infty}^{+\infty} e^{i p\left(\psi-\psi^{\prime}\right)} \int_{0}^{+\infty} v e^{-v(\zeta+z)} J_{p}(v h) J_{p}^{\prime}\left(v h^{\prime}\right) d v \\
& \frac{\partial}{\partial n_{\mathrm{Q}}} G_{w}=2 v_{0} p \cdot v \cdot \sum_{p=-\infty}^{\infty} \int_{0}^{+\infty} v \frac{1}{v-v_{0}} e^{-v(z+\zeta)} e^{i p\left(\varphi-\varphi^{\prime}\right)} J_{p}(v h) J_{p}^{\prime}\left(v h^{\prime}\right) d v \\
& \frac{\partial}{\partial n_{\mathrm{Q}}} G_{c}=i 2 \pi v_{0}^{2} e^{-v_{0}(z+\zeta)} \sum_{p=-\infty}^{\infty} e^{i p\left(\varphi-\varphi^{\prime}\right)} J_{p}\left(v_{0} h\right) J_{p}^{\prime}\left(v_{0} h^{\prime}\right)
\end{aligned}
$$

Similar to the calculation of $G_{n m, k l}, H_{n m, k l}$ can also be divided into the following four parts

$$
\begin{aligned}
& H_{n m, k l 1}=\frac{1}{2 \pi} \int_{0}^{+\infty} \int_{-\pi}^{\pi} \int_{c} \frac{\partial}{\partial n_{\mathrm{Q}}} G_{r} \ell_{k}(\zeta) \ell_{n}(z) e^{i l \varphi^{\prime}} e^{-i m \varphi} d s d \varphi d z \\
& H_{n m, k l 2}=\frac{1}{2 \pi} \int_{0}^{+\infty} \int_{-\pi}^{\pi} \int_{c} \frac{\partial}{\partial n_{\mathrm{Q}}} G_{r^{\prime}} \ell_{k}(\zeta) \ell_{n}(z) e^{i l \varphi^{\prime}} e^{-i m \varphi} d s d \varphi d z \\
& H_{n m, k l 3}=\frac{1}{2 \pi} \int_{0}^{+\infty} \int_{-\pi}^{\pi} \int_{c} \frac{\partial}{\partial n_{\mathrm{Q}}} G_{w} \ell_{k}(\zeta) \ell_{n}(z) e^{i l \varphi^{\prime}} e^{-i m \varphi} d s d \varphi d z \\
& H_{n m, k l 4}=\frac{1}{2 \pi} \int_{0}^{+\infty} \int_{-\pi}^{\pi} \int_{c} \frac{\partial}{\partial n_{\mathrm{Q}}} G_{c} \ell_{k}(\zeta) \ell_{n}(z) e^{i l \varphi^{\prime}} e^{-i m \varphi} d s d \varphi d z
\end{aligned}
$$

The result can be written as

$$
\begin{aligned}
& H_{n m, k l 1}=4 \pi h \sum_{p=-\infty}^{+\infty} \int_{0}^{+\infty} v J_{p}(v h) J_{p}^{\prime}(v h) \frac{2}{2 v+1} d v \cdot \delta_{p m} \delta_{p l} \quad \text { for } n=k \\
& H_{n m, k l 1}=2 \pi h \sum_{p=-\infty}^{+\infty} \int_{0}^{+\infty} v J_{p}(v h) J_{p}^{\prime}(v h) \frac{-4}{(2 v-1)(2 v+1)}\left(\frac{2 v-1}{2 v+1}\right)^{|k-n|} d v \cdot \delta_{p m} \delta_{p l} \text { for } n \neq k \\
& H_{n m k l 2}=8 \pi h \sum_{p=-\infty}^{+\infty} \delta_{p m} \delta_{p l} \int_{0}^{+\infty} v J_{p}(v h) J_{p}^{\prime}(v h) \frac{(2 v-1)^{n+k}}{(2 v+1)^{n+k+2}} d v \\
& H_{n m k l 3}=16 \pi h v_{0} \delta_{p l} \delta_{p m} \sum_{p=-\infty}^{\infty} p \cdot v \cdot \int_{0}^{+\infty} J_{p}(v h) J_{p}^{\prime}(v h) \frac{v}{v-v_{0}} \frac{(2 v-1)^{n+k}}{(2 v+1)^{n+k+2}} d v \\
& H_{n m k l 4}=16 \pi^{2} h^{\prime} v_{0}^{2} i \sum_{p=-\infty}^{+\infty} \delta_{p m} \delta_{p l} \cdot J_{p}\left(v_{0} h\right) J_{p}^{\prime}\left(v_{0} h^{\prime}\right) \frac{\left(2 v_{0}-1\right)^{n+k}}{\left(2 v_{0}+1\right)^{n+k+2}}
\end{aligned}
$$


In the extended boundary integral equation method, when the field point is on the control surface and the source point on the interior free surface, the normal derivative of Kelvin source on the free surface can be written as

$$
\begin{aligned}
& \frac{\partial}{\partial n_{\mathrm{Q}}} G=-\frac{\partial}{\partial \zeta} G=-\frac{\partial}{\partial \zeta}\left(G_{r}+G_{r^{\prime}}+G_{w}+G_{c}\right) \\
& -\frac{\partial}{\partial \zeta}\left(G_{r}+G_{r^{\prime}}\right)=0 \\
& \frac{\partial}{\partial n_{\mathrm{Q}}} G_{w}=2 v_{0} p \cdot v \cdot \sum_{p=-\infty}^{\infty} \int_{0}^{+\infty} \frac{v}{v-v_{0}} e^{-v z} e^{i p\left(\varphi-\varphi^{\prime}\right)} J_{p}(v h) J_{p}\left(v h^{\prime}\right) d v \\
& \frac{\partial}{\partial n_{\mathrm{Q}}} G_{c}=i 2 \pi v_{0}^{2} e^{-v_{0} z} \sum_{p=-\infty}^{\infty} e^{i p\left(\varphi-\varphi^{\prime}\right)} J_{p}\left(v_{0} h\right) J_{p}\left(v_{0} h^{\prime}\right)
\end{aligned}
$$

$H_{n m, F}$ defined in Eq. (27) can be divided into four parts

$$
H_{n m, F}=H_{n m, F 1}+H_{n m, F 2}+H_{n m, F 3}+H_{n m, F 4}
$$

where

$$
\begin{aligned}
& H_{n m, F 1}+H_{n m, F 2}=0 \\
& H_{n m, F 3}=\frac{1}{2 \pi} \int_{0}^{+\infty} \int_{-\pi}^{\pi} \int_{\Delta F_{i}} \frac{\partial G_{w}}{\partial n_{\mathrm{Q}}} \ell_{n}(z) e^{-i m \varphi} d s d \varphi d z \\
& H_{n m, F 4}=\frac{1}{2 \pi} \int_{0}^{+\infty} \int_{-\pi}^{\pi} \int_{\Delta F_{i}} \frac{\partial G_{c}}{\partial n_{\mathrm{Q}}} \ell_{n}(z) e^{-i m \varphi} d s d \varphi d z
\end{aligned}
$$

Substitute Eq. (3.28) into Eq. (3.32)

$$
H_{n m, F 3}=2 v_{0} \Delta S_{F_{i}} e^{-i m \varphi^{\prime}} \int_{0}^{+\infty} \frac{v}{v-v_{0}} \frac{2(2 v-1)^{n}}{(2 v+1)^{n+1}} J_{m}(v h) J_{m}\left(v h^{\prime}\right) d v
$$

Substitute Eq. (3.29) into Eq. (3.33)

$$
H_{n m, F 4}=\frac{4 \pi\left(2 v_{0}-1\right)^{n}}{\left(2 v_{0}+1\right)^{n+1}} v_{0}^{2} J_{m}\left(v_{0} h\right) J_{m}\left(v_{0} h^{\prime}\right)_{\Delta} S_{F_{i}} e^{i m\left(-\varphi^{\prime}\right)_{i}}
$$

When the field point is on the free surface and the source point on the control surface, $G_{F, k l}$ defined in Eq. (30) is divided into four parts

$$
\begin{aligned}
G_{F, k l} & =G_{F, k l 1}+G_{F, k l 2}+G_{F, k l 3}+G_{F, k l 4} \\
G_{F, k l 1} & =\int_{c} G_{r} \ell_{k}(\zeta) e^{i l \varphi^{\prime}} d s
\end{aligned}
$$




$$
\begin{aligned}
G_{F, k l 2} & =\int_{c} G_{r^{\prime}} \ell_{k}(\zeta) e^{i l \varphi^{\prime}} d s \\
G_{F, k l 3} & =\int_{c} G_{w^{\prime}} \ell_{k}(\zeta) e^{i l \varphi^{\prime}} d s \\
G_{F, k l 4} & =\int_{c} G_{c} \ell_{k}(\zeta) e^{i l \varphi^{\prime}} d s
\end{aligned}
$$

Among them, $G_{F, k l 1}=G_{F, k l 2}$, and the derivation of $G_{F, k l 1}$ is given in Eq. (4.4) in Appendix 4,

$$
\begin{aligned}
& G_{F, k l 3}=4 \pi h^{\prime} v_{0} e^{i l \varphi} \int_{0}^{+\infty} \frac{1}{v-v_{0}} \frac{2(2 v-1)^{k}}{(2 v+1)^{k+1}} J_{l}(v h) J_{l}\left(v h^{\prime}\right) d v \\
& G_{F, k l 4}=8 \pi^{2} v_{0} h^{\prime} e^{i l \varphi} \frac{\left(2 v_{0}-1\right)^{k}}{\left(2 v_{0}+1\right)^{k+1}} J_{l}\left(v_{0} h\right) J_{l}\left(v_{0} h^{\prime}\right)
\end{aligned}
$$

In a similar way, $H_{F, k l}$ defined in Eq. (29) can also be divided into four parts, and $H_{F, k l 1}=H_{F, k l 2}$, and the result of $H_{F, k l 1}$ will be given in Eq. (4.5) of Appendix 4, referring to Eq. (3.15) and Eq. (3.16), then one can obtain the following results

$$
\begin{gathered}
H_{F, k l 3}=8 \pi v_{0} h^{\prime} e^{i l \varphi} p \cdot v \cdot \int_{0}^{+\infty} \frac{v}{v-v_{0}} \frac{(2 v-1)^{k}}{(2 v+1)^{k+1}} J_{l}(v h) J_{l}^{\prime}\left(v h^{\prime}\right) d v \\
H_{F, k l 4}=8 \pi^{2} v_{0}^{2} h^{\prime} e^{i p \varphi} J_{l}\left(v_{0} h\right) J_{l}^{\prime}\left(v_{0} h^{\prime}\right) \frac{\left(2 v_{0}-1\right)^{k}}{\left(2 v_{0}+1\right)^{k+1}}
\end{gathered}
$$

When both the field point and source point are on the interior free surface, similar to the way of dealing with $H_{n m, k l}, H_{F, F}$ defined in Eq. (31) can be divided into four parts, and it is obviously that $H_{F, F 1}=0, H_{F, F 2}=0$,

$$
\begin{aligned}
& H_{F, F 3}=\int_{\Delta F_{i}} \frac{\partial}{\partial n_{\mathrm{Q}}} G_{w} d s=2 v_{0} p \cdot v \cdot \int_{0}^{+\infty} \frac{1}{v-v_{0}} e^{-v z} v J_{0}(v R) d v \cdot \Delta S_{F_{i}} \\
& H_{F, F 4}=\int_{\Delta F_{i}} \frac{\partial}{\partial n_{\mathrm{Q}}} G_{c} d s=\int_{\Delta F_{i}}\left[2 \pi v_{0}^{2} J_{0}\left(v_{0} R\right)\right] d s=2 \pi v_{0}^{2} J_{0}\left(v_{0} R\right) \cdot \Delta S_{F_{i}}
\end{aligned}
$$

\section{APPENDIX 4}

The calculation of the integrals defined in Eqs. (50) and (55).

$G_{r, n m, k l}, H_{r, n m, k l}$ are the first parts of $G_{n m, k l}, H_{n m, k l}$ respectively, which have been solved in Appendix 3, when the source point Q is on the body surface or on the free surface, the source on each panel is constant. 


$$
\begin{aligned}
& G_{B, n m, \alpha}=\frac{1}{2 \pi} \int_{0}^{+\infty} \int_{-\pi}^{\pi} \int_{B^{\alpha}} G_{r} \ell_{n}(z) e^{-i m \varphi} d s d \varphi d z \\
& =e^{-i m \varphi^{\prime}} \Delta S_{\alpha} \int_{0}^{+\infty} J_{m}(v h) J_{m}\left(v h^{\prime}\right) d v\left[\int_{0}^{\zeta} e^{-v(\zeta-z)} \ell_{n}(z) d z+\int_{\zeta}^{+\infty} e^{-v(z-\zeta)} \ell_{n}(z) d z\right]
\end{aligned}
$$

where $\Delta S_{\alpha}$ represents the area of panel on the body surface. Substitute the result of 1.1 and 1.2 in Appendix 1 into Eq. (4.1), then $G_{B, n m, \alpha}$ can be solved for.

The normal derivative of Kelvin source on the body surface can be written as

$$
\frac{\partial}{\partial n_{\mathrm{Q}}} G=\frac{\partial}{\partial h^{\prime}} G\left(\cos \varphi^{\prime} n_{1}+\sin \varphi^{\prime} n_{2}\right)+\frac{1}{h^{\prime}} \frac{\partial}{\partial \varphi^{\prime}} G\left(\cos \varphi^{\prime} n_{2}-\sin \varphi^{\prime} n_{1}\right)+\frac{\partial}{\partial \zeta} G \cdot n_{3}
$$

then

$$
\begin{aligned}
& H_{B, n m, \alpha}=\frac{1}{2 \pi} \int_{0}^{+\infty} \int_{-\pi}^{\pi} \int_{B^{\alpha}} \frac{\partial}{\partial n_{\mathrm{Q}}} G_{r} \ell_{n}(z) e^{-i m \varphi} d s d \varphi d z \\
& =\Delta S_{B^{\alpha}} e^{-i m \varphi^{\prime}}\left(\cos \varphi^{\prime} n_{1}+\sin \varphi^{\prime} n_{2}\right) \int_{0}^{+\infty} v J_{m}(v h) J_{m}^{\prime}\left(v h^{\prime}\right) d \kappa \int_{0}^{+\infty} e^{-v|\zeta-z|} \ell_{n}(z) d z \\
& +\Delta S_{B^{\alpha}} e^{-i m \varphi^{\prime}} \frac{(-i m)}{h^{\prime}}\left(\cos \varphi^{\prime} n_{2}-\sin \varphi^{\prime} n_{1}\right) \int_{0}^{+\infty} J_{m}(v h) J_{m}\left(v h^{\prime}\right) d v \int_{0}^{+\infty} e^{-v|\zeta-z|_{n}} \ell_{n}(z) d z \\
& +\Delta S_{B^{\alpha}} e^{-i m \varphi^{\prime}} \int_{0}^{+\infty} v J_{m}(v h) J_{m}\left(v h^{\prime}\right) d v \int_{0}^{+\infty} e^{-v|\zeta-z|_{n}}(z) d z
\end{aligned}
$$

When the source point $\mathrm{Q}$ is on the free surface, $\zeta=0$

$$
\begin{aligned}
& G_{F, n m, \beta}=e^{-i m \varphi^{\prime}} \Delta S_{\beta} \int_{0}^{+\infty} J_{m}(v h) J_{m}\left(v h^{\prime}\right)\left(\frac{2 v-1}{2 v+1}\right)^{n} \frac{2}{2 v+1} d v \\
& H_{F, n m, \beta}=e^{-i m \varphi^{\prime}} \Delta S_{F^{\beta}} \int_{0}^{+\infty} \frac{2 v}{2 v+1} \frac{(2 v-1)^{n}}{(2 v+1)^{n}} J_{m}(v h) J_{m}\left(v h^{\prime}\right) d v
\end{aligned}
$$

When the field point is on the body surface and the source point on the control surface

$$
\begin{aligned}
G_{c, \gamma, k l} & =\int_{c} G_{r} \ell_{k}(\zeta) e^{i l \varphi^{\prime}} d s \\
& =\int_{c}\left[\sum_{p=-\infty}^{+\infty} e^{i p\left(\varphi-\varphi^{\prime}\right)} \int_{0}^{+\infty} e^{-v|\zeta-z|} J_{p}(v h) J_{p}\left(v h^{\prime}\right) d v\right] \ell_{k}(\zeta) e^{i l \varphi^{\prime}} d s \\
& =2 \pi e^{i l \varphi} h^{\prime} \int_{0}^{+\infty} J_{l}(v h) J_{l}\left(v h^{\prime}\right) d v \int_{0}^{+\infty} e^{-v|\zeta-z|} d \zeta \\
H_{c, \gamma, k l} & =\int_{c} \frac{\partial}{\partial n_{\mathrm{Q}}} G_{r} \ell_{k}(\zeta) e^{i l \varphi^{\prime}} d s \\
& =2 \pi h^{\prime} e^{i l \varphi} \int_{0}^{+\infty} v J_{l}(v h) J_{l}^{\prime}\left(v h^{\prime}\right) d \kappa \int_{0}^{+\infty} e^{-v|\zeta-z|} d \zeta
\end{aligned}
$$

When the field point is on the free surface and the source point is the control surface 


$$
\begin{aligned}
& G_{c, \gamma, k l}=2 \pi h^{\prime} e^{i l \varphi} \int_{0}^{+\infty} J_{l}(v h) J\left(v h^{\prime}\right) \frac{2}{2 v+1}\left(\frac{2 v-1}{2 v+1}\right)^{k} d v \\
& H_{c, \gamma, k l}=2 \pi h^{\prime} e^{i l \varphi} \int_{0}^{+\infty} v J_{l}(v h) J_{l}^{\prime}\left(v h^{\prime}\right) \frac{2}{2 v+1}\left(\frac{2 v-1}{2 v+1}\right)^{k} d v
\end{aligned}
$$

Corresponding author: Hui Li, College of Shipbuilding Engineering, Harbin Engineering University, Heilongjiang 150001,China. E-mail: Huili@ hrbeu.edu.cn, Tel.: +86 13936279332.

Submitted: $\quad$ 12.08.2015. Accepted: 24.07.2016.
Hui Li, huili@hrbeu.edu.cn

Lizhu Hao

Huilong Ren

Bo Tian

College of Shipbuilding Engineering, Harbin Engineering University, Heilongjiang 150001, China. 